\title{
On the Modular Operator of Mutli-component Regions in Chiral CFT
}

\author{
Stefan Hollands \\ Institut für Theoretische Physik, Universität Leipzig, Brüderstrasse 16, 04103 Leipzig, Germany. \\ E-mail: stefan.hollands@uni-leipzig.de
}

Received: 5 January 2020 / Accepted: 26 February 2021

Published online: 29 April 2021 - (C) The Author(s) 2021

\begin{abstract}
We introduce a new approach to find the Tomita-Takesaki modular flow for multi-component regions in general chiral conformal field theory. Our method is based on locality and analyticity of primary fields as well as the so-called Kubo-MartinSchwinger (KMS) condition. These features can be used to transform the problem to a Riemann-Hilbert problem on a covering of the complex plane cut along the regions, which is equivalent to an integral equation for the matrix elements of the modular Hamiltonian. Examples are considered.
\end{abstract}

\section{Introduction}

The reduced density matrix of a subsystem induces an intrinsic internal dynamics called the "modular flow". The flow is non-trivial only for non-commuting observable algebras-i.e., in quantum theory-and depends on both the subsystem and the given state of the total system. It has been subject to much attention in theoretical physics in recent times because it is closely related to information theoretic concepts. As examples for some topics such as Bekenstein bounds, Quantum Focussing Conjecture, c-theorems, holography we mention [1-5]. In mathematics, the modular flow has played an important role in the study of operator algebras through the work of Connes, Takesaki and others, see [6] for an encyclopedic account.

It has been known almost from the beginning that the modular flow has a geometric nature in local quantum field theory when the subsystem is defined by a spacetime region of a simple shape such as an interval in chiral conformal field theory (CFT) [7-9]: it is the 1-parameter group of Möbius transformations leaving the interval fixed. For more complicated regions, important progress was made only much later in a pioneering work by Casini et al. [10], who were able to determine the flow for multi-component regions for a chiral half of free massless fermions in two dimensions. Recently in [11] they have generalized their method to the conformal theory of a chiral $U(1)$-current. Unfortunately, the method by $[10,11]$, as well as all other concrete methods known to the author, is based 
in an essential way on special properties of free quantum field theories. The purpose of this paper is to develop methods that could give a handle on the problem in general chiral CFTs, i.e. the left-moving half of a CFT on a (compactified) lightray in $1+1$ dimensional Minkowski spacetime, and to make some of the constructions in the literature rigorous by our alternative method.

Consider a (possibly mixed) state in the chiral CFT, described by a density matrix $\rho$. Typical states of interest are the vacuum $\rho=\left|\Omega_{0}\right\rangle\left\langle\Omega_{0}\right|$, or a thermal state $\rho=$ $e^{-\beta L_{0}} / \operatorname{Tr} e^{-\beta L_{0}}$. Given a union $A=\cup_{j}\left(a_{j}, b_{j}\right)$ of intervals of the (compactified) lightray, we can consider its reduced density matrix $\operatorname{Tr}_{A^{\prime}} \rho=\rho_{A}$, where $A^{\prime}$ is the complement of $A$. For the purposes of this discussion, we restrict to the vacuum state, although in the main part, thermal states will play a major role as well. If $\phi(x)$ is a primary field localized at $x \in A$, the "modular flow" is the Heisenberg time evolution $\rho_{A}^{i t} \phi(x) \rho_{A}^{-i t}$. The object $\rho_{A}$ is not actually well-defined in quantum field theory, but the modular flow is. Below we will use the rigorous framework of Tomita-Takesaki theory in our construction, but for pedagogical purposes, we here pretend that $\rho_{A}$ exists. Formally, the Hilbert space $\mathcal{H}$ splits as $\mathcal{H}_{A} \otimes \mathcal{H}_{A^{\prime}}$ and if $\rho$ is pure, then $\rho_{A}$ is formally a density matrix on $\mathcal{H}_{A}$. Its - equally formal-logarithm $H_{A}=\ln \rho_{A}$ is called the modular Hamiltonian in the physics literature.

In mathematical terms, the quantity which is well defined is the operator $\Delta=\rho_{A} \otimes$ $\rho_{A^{\prime}}^{-1}$. For $x \in A$, we can then also write $\rho_{A}^{i t} \phi(x) \rho_{A}^{-i t}=\Delta^{i t} \phi(x) \Delta^{-i t}$ and $\ln \Delta=$ $H_{A} \otimes 1_{A^{\prime}}-1_{A} \otimes H_{A^{\prime}}$. Furthermore, one can write

$$
\operatorname{Tr}\left(\phi(x) \rho_{A}^{i t} \phi(y) \rho_{A}^{1-i t}\right)=\left\langle\Omega_{0} \mid \phi(x) \Delta^{i t} \phi(y) \Omega_{0}\right\rangle,
$$

and since the conformal primaries generate the full Hilbert space (mathematically, the Reeh-Schlieder theorem), we see that we knowledge of this quantity for all primaries $\phi$ suffices, in principle, to determine all matrix elements of $\Delta^{i t}$, hence the operator itself, hence the flow. Alternatively, to know the generator of the flow, it suffices to know $\left\langle\Omega_{0} \mid \phi(x)(\ln \Delta) \phi(y) \Omega_{0}\right\rangle$. It is those types of quantities which we will study in this paper.

Our main innovation is the following trick and it variants. For $s>0$ and fixed $y \in A$, define a function of $x$ on the complex plane cut along the intervals $A$,

$$
F(s, x, y)= \begin{cases}\left\langle\Omega_{0} \mid \phi(x)\left[1-e^{s}(1-\Delta)^{-1}\right]^{-1} \phi(y) \Omega_{0}\right\rangle & \text { if } \Im(x)<0, \\ \left\langle\Omega_{0} \mid \phi(y)\left[1-e^{s}\left(1-\Delta^{-1}\right)^{-1}\right]^{-1} \phi(x) \Omega_{0}\right\rangle & \text { if } \Im(x)>0 .\end{cases}
$$

Then not only do the usual properties of CFTs imply that this function is holomorphic on the mutliply cut plane, but we also know its jumps across the cuts, given by the functional equation

$$
\left(1-e^{s}\right) F(s, x-i 0, y)-F(s, x+i 0, y)=\left\langle\Omega_{0} \mid[\phi(x), \phi(y)] \Omega_{0}\right\rangle .
$$

The commutator on the right side is given by a sum of $\delta$-functions and their derivatives by locality. We also prove certain further general properties of this function such as the degree of divergences as $x$ approaches $y$ or any boundary of a cut which depend on the conformal dimension of $\phi$. Using this functional equation and a standard contour argument appearing frequently in the study of Riemann-Hilbert type problems, we then obtain a linear integral equation for $F$ of Cauchy-type. The desired matrix elements of the modular Hamiltonian are related by the integral

$$
\left\langle\Omega_{0} \mid \phi(x)(\ln \Delta) \phi(y) \Omega_{0}\right\rangle=\int_{0}^{\infty} \mathrm{d} s F(s, x, y) .
$$


A variant of this method also works for fermionic fields and for thermal states where the corresponding function $F$ lives on a torus cut along $A$ and satisfies a corresponding integral equation.

The basis of our method is an old observation in quantum statistical mechanics. Consider a statistical operator $\rho$. The expectation functional acting an observable $X$ is $\omega(X)=\operatorname{Tr}(X \rho)$ and the modular flow acting on an observable $X$ is, by definition, $\sigma^{t}(X)=\rho^{i t} X \rho^{-i t}$. For observables $X, Y$, consider the function $\varphi_{X, Y}(t)=$ $\omega\left(X \sigma^{t}(Y)\right)=\operatorname{Tr}\left(X \rho^{i t} Y \rho^{1-i t}\right)$. Since $\rho$ is a positive operator, one expects this function to be analytic inside the strip $\{t \in \mathbb{C} \mid-1<\Im(t)<0\}$. The values at the two boundaries of the strip are evidently related by the functional equation

$$
\varphi_{X, Y}(t-i)=\varphi_{Y, X}(-t) .
$$

This functional equation is called the "KMS-condition" [12]. In our case, things are set up in such a way that the KMS condition gives (3) and its variants, from which everything else follows.

Our main results which go beyond the existing literature are the integral equations in cors. 1, 2 which in principle give a way to go beyond free bosonic or fermionic CFTs. In the case of free field theories, the explicit formula in Theorem 1 is a new result, as are for instance the (still somewhat implicit) expressions for the modular hamiltonian of free bosons on a torus given in Sect. 5.4. These results, and similar ones for free fermions on the torus in Sect. 5.2 are also important because they relate our general method to a method, valid for free bosons and fermions, due to [13,14], and therefore give full mathematical justification of these general formulas in the case of type III representations studied here.

This paper is organized as follows. In Sects. 2 and 3, we review basic notions from operator algebras, Tomita-Takesaki theory, and the operator algebraic approach to CFT (conformal nets) in order to make the paper self-contained. In Sects. 4 and 5 we introduce our method and study several examples. We conclude in Sect. 6. Some conventions for elliptic functions are described in the appendix.

Notations and conventions: Gothic letters $\mathfrak{A}, \mathfrak{M}, \ldots$ denote $*$-algebras, usually v. Neumann algebras. Calligraphic letters $\mathcal{H}, \mathcal{K}, \ldots$ denote linear spaces, always assumed to be separable. The inverse temperature $\beta$ and modular parameter $\tau$ are related by $-2 \pi i \tau=\beta$. The branches of $\ln z$ and $z^{\alpha}$ are taken along the negative real axis. $\mathbb{S}=\{z \in \mathbb{C}|| z \mid=1\}$ denotes the unit circle, $\mathbb{D}^{ \pm}$its interior/exterior.

Note added in proof: After this preprint was submitted, it was pointed out to us by the authors of [15] that one of our calculations related to thermal states contained an error, creating a tension between some of our results and those by [15], see also [16]. We are grateful to these authors for making us aware of this issue, which has been fixed in the current version.

\section{Review of Modular Theory}

2.1. Modular flow. For the convenience of the unfamiliar reader we review the basic elements of modular (= Tomita-Takesaki-) theory; detailed references are $[6,17,18]$. Connections to quantum information theory are described in [19]. An exposition directed towards a theoretical physics audience is [20].

The notion of modular flow is embedded into the theory of v. Neumann algebras. Such an algebra, $\mathfrak{M}$, can be defined as a complex linear space of bounded operators on 
some Hilbert space ${ }^{1} \mathcal{H}$ that is closed under taking products, adjoints (denoted by $*$ ). Such limits are understood in the so called "weak" topology, i.e. convergence of matrix elements. It is common to denote by $\mathfrak{M}^{\prime}$ the commutant, defined as the set of all bounded operators on $\mathcal{H}$ commuting with all operators in $\mathfrak{M}$.

To define the objects of main interest of the theory, one has to assume that $\mathfrak{M}$ is in "standard form", meaing: $\mathcal{H}$ contains a "cyclic and separating" vector for $\mathfrak{M}$, that is, a unit vector $|\Omega\rangle$ such that the set consisting of $X|\Omega\rangle, X \in \mathfrak{M}$ is a dense subspace of $\mathcal{H}$, and such that $X|\Omega\rangle=0$ implies $X=0$ for any $X \in \mathfrak{M}$. The point is that one can then consistently define the anti-linear Tomita operator $S$ on the domain $\mathcal{D}(S)=\{X|\Omega\rangle \mid X \in \mathfrak{M}\}$ by the formula

$$
S X|\Omega\rangle=X^{*}|\Omega\rangle \text {. }
$$

The cyclic property is needed in order that $S$ is densely defined, whereas without the separating property the definition would not be self-consistent. One can show that $S$ is a closable operator. This technical property guarantees that $S$ has a polar decomposition. It is customarily denoted by $S=J \Delta^{\frac{1}{2}}$, where $J$ anti-linear and unitary and $\Delta$ self-adjoint and non-negative. Tomita-Takesaki theory is about the interplay between the operators $\Delta, J$ and the algebras $\mathfrak{M}, \mathfrak{M}^{\prime}$. The basic theorem is:

(i) $J$ exchanges $\mathfrak{M}$ with the commutant in the sense that $J \mathfrak{M} J=\mathfrak{M}^{\prime}$. Furthermore, $J^{2}=1, J \Delta J=\Delta^{-1}$.

(ii) The modular flow $\sigma^{t}(X)=\Delta^{i t} X \Delta^{-i t}$ leaves $\mathfrak{M}$ and $\mathfrak{M}^{\prime}$ invariant for all $t \in \mathbb{R}$.

(iii) From the vector $|\Omega\rangle$, one can define the state functional $\omega(X)=\langle\Omega \mid X \Omega\rangle, \omega$ : $\mathfrak{M} \rightarrow \mathbb{C}$. It is positive and normalized (meaning $\omega\left(X^{*} X\right) \geqslant 0 \forall X \in \mathfrak{M}, \omega(1)=$ 1 ), and invariant under the modular flow in the sense that $\omega \circ \sigma^{t}=\omega$ for all $t \in \mathbb{R}$. The KMS-condition holds: for all $X, Y \in \mathfrak{M}$, the bounded function

$$
t \mapsto \varphi_{X, Y}(t)=\omega\left(X \sigma^{t}(Y)\right) \equiv\left\langle\Omega \mid X \Delta^{i t} Y \Omega\right\rangle
$$

has an analytic continuation to the strip $\{z \in \mathbb{C} \mid-1<\mathfrak{s} z<0\}$ with the property that its boundary value for $\Im z \rightarrow-1^{+}$exists and is equal to

$$
\varphi_{X, Y}(t-i)=\omega\left(\sigma^{t}(Y) X\right) .
$$

A partial converse to (iii) is: If $\omega^{\prime}$ is a normal (i.e. continuous in the weak*-topology) positive linear functional on $\mathfrak{M}$, then it has a unique vector representative $\left|\Omega^{\prime}\right\rangle$ in the natural cone $\mathcal{P}^{\sharp}:=\{X j(X)|\Omega\rangle \mid X \in \mathfrak{M}\}$, where $j(X)=J X J$; in other words $\omega^{\prime}(X)=\left\langle\Omega^{\prime} \mid X \Omega^{\prime}\right\rangle$ for all $X \in \mathfrak{M}$.

The objects $J, \Delta, \mathcal{P}^{\sharp}$ depend on the algebra $\mathfrak{M}$ and the state $|\Omega\rangle$.

Example 0: Even tough Tomita-Takesaki theory is most interesting in the case of infinite dimensional v. Neumann algebras of types II, III, it helps with intuition to have in mind the finite dimensional case, i.e. the type $\mathrm{I}_{n}$ ' (algebra of $n$ by $n$ matrices). In this case, $\mathfrak{M}=M_{n}(\mathbb{C}) \otimes 1_{n}$, which acts on the Hilbert space $\mathcal{H}=\mathbb{C}^{n} \otimes \mathbb{C}^{n}$. Evidently, the commutant is $\mathfrak{M}^{\prime}=1_{n} \otimes M_{n}(\mathbb{C})$. A vector $|\Omega\rangle$ in this Hilbert space is cyclic and separating if $|\Omega\rangle=\sum_{j=1}^{n} \sqrt{p_{j}}|j\rangle \otimes|j\rangle$ in some ON basis $\{|j\rangle\}$ and iff all $p_{j}>0$, $\sum_{j=1}^{n} p_{j}=1$. The state functional $\omega$ can be written in this example in terms of the "reduced density matrix"

$$
\rho_{\omega}=\sum_{j=1}^{n} p_{j}|j\rangle\langle j|, \quad \omega(X)=\operatorname{Tr}_{\mathbb{C}^{n}}\left(X \rho_{\omega}\right) \quad(X \in \mathfrak{M}) .
$$

\footnotetext{
1 We always assume that $\mathcal{H}$ is separable.
} 
In fact, any positive normalized state functional $\omega^{\prime}$ arises from a unique reduced density matrix $\rho_{\omega^{\prime}}$ in this way. It is easy to go through the definition of $\Delta, J$ via $S$ giving for instance that

$$
\Delta^{\frac{1}{2}}=\rho_{\omega}^{\frac{1}{2}} \otimes \rho_{\omega}^{-\frac{1}{2}}
$$

Therefore, the modular flow is $\sigma^{t}(X)=\rho_{\omega}^{i t} X \rho_{\omega}^{-i t}$. The "modular Hamiltonian" is defined as the self-adjoint operator $\ln \Delta$. In our example, therefore, $\ln \Delta=\ln \rho_{\omega} \otimes 1_{n}-$ $1_{n} \otimes \ln \rho_{\omega}$, where the first term belongs to $\mathfrak{M}$ and the second to $\mathfrak{M}^{\prime}$. It is important to stress that the split of $\ln \Delta$ into a part from $\mathfrak{M}$ and one from $\mathfrak{M}^{\prime}$ is impossible for general v. Neumann algebras, in particular for the type $\mathrm{III}_{1}$-factors appearing in quantum field theories. ${ }^{2}$ Therefore, apart from trivial cases, the object $\ln \rho_{\omega}$, hence the reduced density operator $\rho_{\omega}$ itself, does not exist. On the other and, $\ln \Delta$ and $\omega$ always exist. We will make sure to work with these well-defined objects in our setting.

Sometimes, a state $\omega$ is only given as an abstract (weakly continuous) expectation functional on an abstract ${ }^{3} \mathrm{v}$. Neumann algebra $\mathfrak{M}$. Then one can perform the basic but very important GNS construction in order to obtain a Hilbert space in which the state is represented by a vector.

The starting point of this construction is the simple observation that the algebra $\mathfrak{M}$ itself, as a linear space, always forms a representation $\pi$ by left multiplication, i.e. $\pi(X) Y \equiv X Y$. To equip this representation with a Hilbert space structure, it is natural to define $\langle X \mid Y\rangle=\omega\left(X^{*} Y\right)$, but this will in general lead to non-zero vectors with vanishing norm, unless $\omega$ is separating. Introduce $\mathfrak{J}_{\omega}=\left\{X \in \mathfrak{M} \mid \omega\left(X^{*} X\right)=0\right\}$. By the Cauchy-Schwarz inequality, $\left|\omega\left(X^{*} Y\right)\right| \leqslant \omega\left(X^{*} X\right)^{1 / 2} \omega\left(Y^{*} Y\right)^{1 / 2}$, we have $\mathfrak{J}_{\omega}=$ $\left\{X \in \mathfrak{M} \mid \forall Y \in \mathfrak{M}, \omega\left(Y^{*} X\right)=0\right\}$, so it is a closed linear subspace and a left ideal of $\mathfrak{M}$ containing precisely the null vectors. We can then define $\mathcal{H}_{\omega}=\mathfrak{M} / \mathfrak{J} \omega$ and complete it in the induced inner product. The left representation induces a representation on $\mathcal{H}_{\omega}$ which is called $\pi_{\omega}$. It is the desired GNS-representation. The vector $\left|\Omega_{\omega}\right\rangle \in \mathcal{H}_{\omega}$ representing $\omega$ is simply the equivalence class of the unit operator, 1 . It is by construction "cyclic" in the sense that the set $\pi_{\omega}(\mathfrak{M})\left|\Omega_{\omega}\right\rangle$ is dense in $\mathcal{H}_{\omega}$. The vector is standard if $\omega\left(X^{*} X\right)$ implies $X=0$ (meaning $\mathfrak{J}_{\omega}=\{0\}$ ), in which case we say that it is faithful.

\section{Review of Chiral CFTs}

3.1. Conformal nets on the real line (lightray). One way to formalize the structure of chiral conformal quantum field theories (CFTs) is via nets of operator algebras. A chiral conformal field theory is associated with one lightray. It is given abstractly by an assignment of an algebra of operators $\mathfrak{A}(I)$ with each open interval $I=(a, b) \subset \mathbb{R}$ of the this lightray.

This assignment is called a conformal net if it obeys the following rules (see [21] for a general introdution to algebraic quantum field theory and e.g. [22,23] for conformal nets):

a1) (Isotony) The algebras $\mathfrak{A}(I)$ are v. Neumann algebras acting on a common Hilbert space $\mathcal{H}$. If $I \subset J$ are intervals, then $\mathfrak{A}(I) \subset \mathfrak{A}(J)$.

\footnotetext{
2 The possibility of making the split implies that $\sigma^{t}$ is inner, i.e. can be written as $\sigma^{t}(X)=U(t) X U(t)^{*}$ for unitaries $U(t)$ in $\mathfrak{M}$. One characterization of type $I I I \mathrm{v}$. Neumann algebras is that $\sigma^{t}$ precisely cannot be inner for any normal state $\omega$.

3 We mean a $C^{*}$-algebra with a preferred "folium" of normal states, see [6]. In particular, it is not assumed that $\mathfrak{M}$ is a priori represented by bounded linear operators on some Hilbert space.
} 
a2) (Causality) Setting $I^{\prime}=\mathbb{R} \backslash[a, b]$ if $I=(a, b)$, we have $\mathfrak{A}\left(I^{\prime}\right) \subset \mathfrak{A}(I)^{\prime}$, i.e. observables from disjoint intervals commute.

a3) (Covariance) On $\mathcal{H}$, there is a unitary representation $g \mapsto U(g)$ of the group $\operatorname{SL}(2, \mathbb{R}) /\{ \pm 1\}$. If we let elements $g=\left(\begin{array}{ll}a & b \\ c & d\end{array}\right)$ of this group act locally on $\mathbb{R}$ by fractional transformations $g(x)=\frac{a x+b}{d x+c}$, then it is assumed that $U(g) \mathfrak{A}(I) U(g)^{*}=$ $\mathfrak{A}(g(I))$ for all intervals $I$ and $g \in \mathrm{SL}(2, \mathbb{R})$ such that $g(x)$ is well defined for all $x \in I$. We also use the notation

$$
\alpha^{g}(X)=\operatorname{Ad}_{U(g)}(X) \equiv U(g) X U(g)^{*} .
$$

a4) (Spectrum) The representation $g \mapsto U(g)$ is strongly continuous. The infinitesimal generator $P$ of translations $\operatorname{tra}_{t}(x)=x+t$, i.e. $P=-\left.i \frac{\mathrm{d}}{\mathrm{d} t} U\left(\operatorname{tra}_{t}\right)\right|_{t=0}$ has nonnegative spectrum.

a5) (Vacuum) There is a unique (unit) vector $\left|\Omega_{0}\right\rangle \in \mathcal{H}$ such that $U(g)\left|\Omega_{0}\right\rangle=\left|\Omega_{0}\right\rangle$. The corresponding state functional will be called $\omega_{0}(X)=\left\langle\Omega_{0} \mid X \Omega_{0}\right\rangle$ throughout. The vacuum should be cyclic for $\bigvee_{I} \mathfrak{A}(I)$, the v. Neumann algebra generated by all intervals.

The algebra of observables associated with the union of $p$ open intervals with disjoint closures,

$$
A=\bigcup_{j=1}^{p}\left(a_{j}, b_{j}\right) \subset \mathbb{R} \quad \text { or } \mathbb{S} \text { below, }
$$

where each $I_{j}=\left(a_{j}, b_{j}\right)$ is an interval of $\mathbb{R}$ (or arc of the circle $\mathbb{S}$ below), is defined to be

$$
\mathfrak{A}(A)=\bigvee_{i=1}^{p} \mathfrak{A}\left(I_{i}\right),
$$

where the symbol $\vee$ means the $\mathrm{v}$. Neumann algebra that is generated by the algebras for the individual arcs/intervals.

3.2. Conformal nets on the circle (compactified lightray). If we want to insist on a global action of the Möbuis group $\operatorname{SL}(2, \mathbb{R}) /\{ \pm 1\}$ on the net, we must pass from the light ray to a compactified lightray, i.e. the circle. The compactification proceeds via the Caley transformation $C: \mathbb{S} \backslash\{+1\} \rightarrow \mathbb{R}, C(x)=-i(x+1) /(x-1)$, and under this transformation intervals get mapped to arcs of the circle. The Caley transform intertwines the action of $\operatorname{SL}(2, \mathbb{R}) /\{ \pm 1\}$ on the lightray with the action $z \mapsto g(z)=\frac{\alpha z+\beta}{\bar{\beta} z+\bar{\alpha}}$ of $\mathrm{SU}(1,1) /\{ \pm 1\}$ on the circle, where $g$ now corresponds to the matrix $\left(\begin{array}{l}\alpha \\ \bar{\beta} \\ \bar{\alpha}\end{array}\right) \in \mathrm{SU}(1,1)$ under the standard isomorphism between the groups $\operatorname{SL}(2, \mathbb{R})$ and $\operatorname{SU}(1,1)$.

The axioms for a conformal field theory, i.e. net of operator algebras, on the circle are completely analogous to those for the lightray. In the circle picture, it is more standard and natural to use the generators of $\operatorname{SU}(1,1)$ called $L_{0}, L_{ \pm 1}$, where $L_{0}$ is the generator of rotations $z \rightarrow e^{i t} z, t \in \mathbb{R}$. The requirement a4) is equivalent to the requirement that $L_{0}$ has non-negative spectrum. From a net on the circle, we may via the Caley 
transform always get a net on the lightray such that $P$ has non-negative spectrum, but not necessarily vice versa since the point at infinity is missing from the lightray. For the rest of the paper, we will assume the axioms on the circle. In Sect. 5, we will also need:

a6) (Finite trace) $\operatorname{Tr} e^{-\beta L_{0}}<\infty$ for $\beta>0$.

The above axioms (including the trace condition just mentioned) have a number of well-known consequences which are of interest for this paper:

1. For each interval $\mathfrak{A}(I)^{\prime}=\mathfrak{A}\left(I^{\prime}\right)$ (Haag duality [9]).

2. For each interval, the linear subspace $\mathfrak{A}(I)\left|\Omega_{0}\right\rangle$ is dense in $\mathcal{H}$ (Reeh-Schlieder theorem). As a consequence, the vector $\left|\Omega_{0}\right\rangle$ is cyclic and separating for each local algebra $\mathfrak{A}(I)$, and we can apply Tomita-Takesaki theory to the pair $\left(\mathfrak{A}(I),\left|\Omega_{0}\right\rangle\right)$.

3. The modular operator $\Delta$ associated with an open arc $I=(a, b)$ acts geometrically in the sense that

$$
\Delta^{i t}=U\left(g_{t}\right), \quad g_{t}(z)=\frac{a(b-z) e^{-2 \pi t}+b(z-a)}{(b-z) e^{-2 \pi t}+(z-a)},
$$

(Hislop-Longo-theorem [9]).

4. Each algebra $\mathfrak{A}(I)$ has in its central decomposition only hyperfinite type $\mathrm{III}_{1}$ factors $[24,25]$.

5. $\overline{\bigcup_{I} \mathfrak{A}(I)}=\mathfrak{B}(\mathcal{H})$ (irreducibility [26]).

Most of these axioms and results have a more or less obvious counterpart for graded local, i.e. "fermionic", theories, see e.g. [27].

Example 4: (Virasoro-net) The Virasoro algebra is the Lie-algebra with generators $\left\{L_{n}, \kappa\right\}_{n \in \mathbb{Z}}$ obeying

$$
\left[L_{n}, L_{m}\right]=(n-m) L_{n+m}+\frac{\kappa}{12} n\left(n^{2}-1\right) \delta_{n,-m}, \quad\left[L_{n}, \kappa\right]=0 .
$$

A positive energy representation on a Hilbert space $\mathcal{H}$ is a representation such that (i) $L_{n}^{*}=L_{-n}$ (unitarity), (ii) $L_{0}$ is diagonalizable with non-negative eigenvalues, and (iii) the central element is represented by $\kappa=c 1$. From now, we assume a positive energy representation. We assume that $\mathcal{H}$ contains a vacuum vector $\left|\Omega_{0}\right\rangle$ which is annihilated by $L_{-1}, L_{0}, L_{1},(\mathfrak{s l}(2, \mathbb{R})$-invariance) and which is a highest weight vector (of weight $0)$, i.e. $L_{n}\left|\Omega_{0}\right\rangle=0$ for all $n>0$. One has the bound [23,28-30]

$$
\left\|\left(1+L_{0}\right)^{k} L_{n} \Psi\right\| \leqslant \sqrt{c / 2}(|n|+1)^{k+3 / 2}\left\|\left(1+L_{0}\right)^{k+1} \Psi\right\|
$$

for $|\Psi\rangle \in \mathcal{V} \equiv \bigcap_{k \geqslant 0} \mathcal{D}\left(L_{0}^{k}\right) \subset \mathcal{H}$ and any natural number $k$.

One next defines from the Virasoro algebra the stress tensor on the unit circle $\mathbb{S}$, identified with points $z=e^{2 \pi i u}, u \in \mathbb{R}$ in $\mathbb{C}$. The stress tensor is an operator valued distribution on $\mathcal{H}$ defined in the sense of distributions by the series

$$
T(z)=-\frac{1}{2 \pi} \sum_{n=-\infty}^{\infty} L_{n} z^{-n-2} .
$$

More precisely, for a test function $f \in C^{\infty}(\mathbb{S})$ on the circle, it follows from (16) that the corresponding smeared field

$$
T(f)=\int_{\mathbb{S}} T(z) f(z) \mathrm{d} z:=-\frac{1}{2 \pi} \sum_{n=-\infty}^{\infty}\left(\int_{\mathbb{S}} z^{-n-2} f(z) \mathrm{d} z\right) L_{n}
$$


is an operator defined e.g. on the dense invariant domain $\mathcal{V}=\bigcap_{k \geqslant 0} \mathcal{D}\left(L_{0}^{k}\right) \subset \mathcal{H}$ (which can be shown to be a common core for the operators $T(f)$ ) and the assignment $f \mapsto T(f)|\psi\rangle$ is continuous in the topologies on $C^{\infty}(\mathbb{S})$ and $\mathcal{H}$ for any vector in this domain. Letting $\Gamma$ be the anti-linear involution

$$
\Gamma f(z)=-z^{2} \overline{f(z)}
$$

the smeared stress tensor is a self-adjoint operator on $\mathcal{D}\left(L_{0}\right)$ for $f$ obeying the reality condition $\Gamma f=f$, and one has $T(f)^{*}=T(\Gamma f)$ in general. It can be shown that the operators $e^{i T(f)}$ for real $f$ form a unitary projective representation of the (covering of the) group of orientation preserving diffeomorphisms (whose generators are the vector fields $f(z) \mathrm{d} / \mathrm{d} z$ ) on the circle. The Virasoro net is then defined by

$$
\mathfrak{A}_{\mathrm{Vir}}(I)=\left\{e^{i T(f)} \mid f \in C^{\infty}(I), \Gamma f=f\right\}^{\prime \prime}
$$

where the double prime means the v. Neumann closure. The generators of this algebra hence correspond to diffeomorphisms acting trivially outside the arc $I \subset \mathbb{S}$.

3.3. Pointlike fields. The standard setup of CFT commonly used in the physics literature is based on the use of pointlike fields rather than nets of algebras of bounded operators. Here we will sketch the connection. In fact, the full mathematical details of this connection are not understood in general, although in many important classes of examples, see [23].

On the circle, one typically postulates the existence of local fields having the "mode expansions"

$$
\phi(z)=\frac{1}{\sqrt{2 \pi}} \sum_{n \in \mathbb{Z}} \phi_{n} z^{-n-h}
$$

$h>0$ is called the conformal dimension of the field. The field is typically "energy bounded" i.e. that the modes $\phi_{n}, n \in \mathbb{Z}$ of the field are linear operators on $\mathcal{H}_{0}$, which satisfy:

Assumption 1. The local fields have a mode expansion (21) such that:

1. an energy bound of the type $\left\|\left(1+L_{0}\right)^{k} \phi_{n} \Psi\right\| \leqslant C(1+|n|)^{k+h-\frac{1}{2}}\left\|\left(1+L_{0}\right)^{k+h-1} \Psi\right\|$ for all $n \in \mathbb{N}_{0},|\Psi\rangle \in \mathcal{H}_{0}$, and for some $k \geqslant 0$, satisfying

2. the commutation relations $\left[L_{m}, \phi_{n}\right]=((h-1) m-n) \phi_{n+m}$ for $|m| \leqslant 1$ where $L_{-1}, L_{0}, L_{1}$ are the generators of the action of $\operatorname{SU}(1,1)$ on $\mathcal{H}_{0}$ and where $h \in \mathbb{R}$ is called the conformal spin, satisfying

3. if $\left|\Omega_{0}\right\rangle \in \mathcal{H}_{0}$ is the vacuum vector, then $\phi_{n}\left|\Omega_{0}\right\rangle=0$ for $n>-h$, and satisfying

4. $\phi_{n}^{*}=\phi_{-n}$ for a self-adjoint local field.

5. The fields $\phi(f), \operatorname{supp}(f) \subset I$ should be affiliated with $\mathfrak{A}(I)$, i.e. there exists a sequence $B_{n}$ such that $\lim _{n} B_{n}|\Psi\rangle=\phi(f)|\Psi\rangle$ for all $\Psi \in \mathcal{V}=\cap_{k} \mathcal{D}\left(L_{0}^{k}\right)$.

These properties imply that the smeared fields are operator valued tempered distributions on the domain $\mathcal{V}=\cap_{k} \mathcal{D}\left(L_{0}^{k}\right)$ : Let $|\Psi\rangle \in \mathcal{V}$. Then 1) gives, with $\phi(f):=$ $\int_{\mathbb{S}} \phi(z) f(z) \mathrm{d} z$ 


$$
\begin{aligned}
\left\|\left(1+L_{0}\right)^{k} \phi(f) \Psi\right\| & \leqslant C\left\|\left(1+L_{0}\right)^{k+h-1} \Psi\right\| \sum_{n \in \mathbb{Z}}\left|\widehat{f}_{-n-h}\right|(1+|n|)^{h-\frac{1}{2}} \\
& \leqslant C_{\Psi} \sup _{j \leqslant h}\left\|f^{(j)}\right\|_{L^{\infty}}
\end{aligned}
$$

because $\left|\widehat{f}_{-n-h}\right|$ goes to zero for $|n| \rightarrow \infty$ faster than any inverse power. Thus, $\phi(f)$ is an operator valued distribution on the dense invariant domain $\mathcal{V}$, which is in fact a common core for the operators $\phi(f)$. By the same type of estimate the properties 1) and 2) imply furthermore that $\phi(z)\left|\Omega_{0}\right\rangle$ can be analytically continued to a $\mathcal{H}$-valued holomorphic function on $\mathbb{D}^{+}$with vector valued distributional boundary value on $\mathbb{S}$. It follows from the commutation relations 2 ) that $\mathcal{H}$ carries a strongly continuous unitary representation $U$ of $\mathrm{SU}(1,1)$ generated by $L_{0}, L_{ \pm 1}$, and this representation satisfies transformation law

$$
\begin{gathered}
i\left[L_{m}, \phi(z)\right]=z^{m+1} \frac{\mathrm{d}}{\mathrm{d} z} \phi(z)+h(\rho) z^{m} \phi(z) \text { or } \\
U(g) \phi(z) U(g)^{*}=\left[g^{\prime}(z)\right]^{h} \phi(g(z)),
\end{gathered}
$$

where $g \in \widehat{\mathrm{SU}(1,1)}$ is in the covering group of the Möbius group, and $g(z)$ its action on points $z$ of the circle. For integer $h \in \mathbb{N}_{0}$, we get a representation of $\mathrm{SU}(1,1) /\{ \pm 1\}$. The restriction of $U$ to the invariant subspace $\operatorname{span}\left\{\phi_{n}\left|\Omega_{0}\right\rangle=0 \mid n \leqslant-h\right\}$ is a discrete series representation (see e.g. IX, para. 3 of [31]). It also follows that primary fields can and will be normalized so that

$$
\left\langle\Omega_{0}|\phi(x) \phi(y)| \Omega_{0}\right\rangle=\frac{e^{-i \pi h}}{2 \pi(x-y)^{2 h}} .
$$

In particular, we see that the field can be local only if the dimension $h$ is a natural number. Fermionic fields are not local but satisfy a graded locality. In that case $h \in \frac{1}{2} \mathbb{N}_{0}$.

Example 5: (Stress tensor) The stress tensor $T(z)$ affiliated with the Virasoro net of central charge $c>0$ is a pointlike field of dimension $h=2$ satisfying the above assumptions.

Example 6: $(U(1)$-current, see e.g. [27,28]) The net of the free $U(1)$ current on the circle can be defined e.g. starting from the Lie-algebra generated by a central element 1 and the "modes" $j_{n}, n \in \mathbb{Z}$ defined by $\left[j_{n}, j_{m}\right]=i n \delta_{n,-m} 1$ with *-operation $j_{n}^{*}=j_{-n}$. The Hilbert space $\mathcal{H}$ is the closure of the linear span of $j_{n_{1}} \ldots j_{n_{k}}\left|\Omega_{0}\right\rangle, n_{1} \leqslant \cdots \leqslant n_{k} \leqslant$ -1 on which the action of the $J_{m}$ 's is obtained via the commutation relations and the condition $j_{n}\left|\Omega_{0}\right\rangle=0$ for $n>-1$. One sets $L_{n}=\frac{1}{2} \sum_{m \in \mathbb{Z}}: j_{n-m} j_{m}:$, where here and in the following, the normal ordering sign : , : means that modes with index $m>-1$ (or $>h$ if the field has dimension $h$ ) are always put to the right of the modes with index $n-m \leqslant-1$. The $L_{n}$ 's the satisfy a Virasoro algebra of central charge $c=1$.

It can be checked that the corresponding current

$$
j(z)=\frac{1}{\sqrt{2 \pi}} \sum_{n \in \mathbb{Z}} j_{n} z^{-n-1}
$$

satisfies the above assumptions with $h=1$ and is hence an operator valued distribution satisfying $j(z)^{*}=z^{2} J(z)$ and $[j(z), j(w)]=i \delta^{\prime}(z-w)$. For any test-function $f$, the smeared operator $j(f):=\int_{\mathbb{S}} j(z) f(z) \mathrm{d} z$ has a dense set of analytic vectors (a space of 
such vectors is spanned by the eigenvectors of $L_{0}$ ), and hence is essentially self-adjoint by Nelson's analytic vector theorem. Hence, we can unambiguously define the Weyl operators

$$
W(f)=e^{i j(f)}, \quad f \in C_{\Gamma}^{\infty}(\mathbb{S}),
$$

(here $\Gamma f(z)=-\overline{f(z)}$ and $C_{\Gamma}^{\infty}(\mathbb{S})$ is the set of invariant elements under $\Gamma$ ), satisfying the Weyl relations

$$
\begin{aligned}
& W(f) W(g)=e^{i C(f, g) / 2} W(f+g), \quad W(f)^{*}=W(-f), \\
& C(f, g)=\frac{1}{2} \int_{\mathbb{S}}\left(g^{\prime} f-f^{\prime} g\right) \mathrm{d} z .
\end{aligned}
$$

The corresponding net of v. Neumann algebras is defined by

$$
\mathfrak{A}_{U(1)}(I) \equiv\left\{W(f) \mid f \in C_{\Gamma}^{\infty}(I)\right\}^{\prime \prime}
$$

where $I \subset \mathbb{S}$ is an open arc of the circle or a union thereof, and the double prime means the weak closure (double commutant). The local, unbounded field operators $j(f)$, supp $f \subset I$ are not contained in- but are affiliated with these algebras.

Example 7: (Free Fermi net, see e.g. [27,32]) This net is constructed starting from the Clifford algebra generated by a central element 1 and the "modes" $\psi_{n}, n \in \mathbb{Z}+\frac{1}{2}$ subject to the relations $\psi_{n} \psi_{m}+\psi_{m} \psi_{n}=\delta_{n,-m} 1, \psi_{n}^{*}=\psi_{-n}$. The vacuum Hilbert space $\mathcal{H}_{\mathrm{NS}}$ is the closure of the linear span of the vectors $\psi_{n_{1}} \cdots \psi_{n_{k}}\left|\Omega_{\mathrm{NS}}\right\rangle, n_{1}<n_{2}<\cdots<0$. A $*$-representation is defined setting $\psi_{n}\left|\Omega_{\mathrm{NS}}\right\rangle=0$ for all $n \geqslant 0$ using the relations to define the action of an arbitrary $\psi_{n}$. The state $\left|\Omega_{\mathrm{NS}}\right\rangle$ is in this context called the "NeveuSchwarz-vacuum". The operators $L_{n}$ are defined by $L_{n}=\sum_{m \in \mathbb{Z}+\frac{1}{2}} m: \psi_{-m+n} \psi_{m}$ : which generate an action of the Virasoro algebra (in particular of the Lie algebra of $\mathrm{SU}(1,1)$ generated by $\left.L_{n}, n=-1,0,1\right)$, at central charge $c=\frac{1}{2}$. The corresponding field

$$
\psi(z)=\frac{1}{\sqrt{2 \pi}} \sum_{n \in \mathbb{Z}} \psi_{n-\frac{1}{2}} z^{-n}
$$

is hence an operator valued distribution. It satisfies $\psi(z)^{*}=z \psi(z)$ and $\psi(z)^{*} \psi(w)+$ $\psi(w) \psi(z)^{*}=\delta(z-w) 1$. For any test-function $f$, the smeared operator $\psi(f):=$ $\int_{\mathbb{S}} \psi(z) f(z) \mathrm{d} z$ is in fact a bounded operator satisfying the canonical anti-commutation relations

$$
\psi(f) \psi(g)+\psi(g) \psi(f)=-(\Gamma f, g) 1, \quad \psi(f)^{*}=\psi(\Gamma f), \quad \Gamma(f)=-z^{-1} \overline{f(z)} .
$$

The corresponding net of v. Neumann algebras is defined as the CAR-algebra [33]

$$
\mathfrak{A}_{\mathrm{Fermi}}(I) \equiv\left\{\psi(f) \mid f \in C_{0}^{\infty}(I)\right\}^{\prime \prime} .
$$

The net of local observables is not a local net but a graded local net, see e.g. [27]. There is another representation of the same net $\mathfrak{A}_{\text {Fermi }}$, called the "Ramond" representation. It is given by the integer moded expansion

$$
\psi(z)=\frac{1}{\sqrt{2 \pi}} \sum_{n \in \mathbb{Z}} \psi_{n} z^{-n-\frac{1}{2}},
$$


where the modes satisfy the same relations as before. The Hilbert space $\mathcal{H}_{\mathrm{R}}$ is constructed as the linear span of the vectors $\psi_{n_{1}} \cdots \psi_{n_{k}}\left|\Omega_{\mathrm{R}}\right\rangle, n_{1}<n_{2}<\cdots \leqslant 0$ setting $\psi_{n}\left|\Omega_{\mathrm{R}}\right\rangle=$ 0 for all $n>0$ using the relations to define the action of an arbitrary $\psi_{n}$. The Virasoro generators in the Ramond representation $\mathcal{H}_{\mathrm{R}}$ are $L_{n}=\sum_{m \in \mathbb{Z}} m: \psi_{-m+n} \psi_{m}:$.

\section{Modular Operators for Conformal Nets on $\mathbb{S}$}

In this section we give a first prescription for computing modular operators of chiral conformal nets on $\mathbb{S}$ satisfying some natural extra conditions. It is related naturally to the matrix elements $\left\langle\Omega_{0}\left|\phi(x) \Delta^{i t} \phi(y)\right| \Omega_{0}\right\rangle$, but leaves in general certain ambiguities that preclude so far their explicit calculation. This difficulty can be overcome to a certain extent by our second method, presented in Sect. 5, more directly related to the matrix element $\langle\Omega|\phi(x)(\ln \Delta) \phi(y)| \Omega\rangle$. Since the material here will form the basis of our discussion in Sect. 5, and since the arguments are also of independent interest, we nevertheless present this approach first.

4.1. General results. Quite generally, if $\mathfrak{M}$ is a v. Neumann algebra in standard form with cyclic and separating vector $\Omega$, then if $X, Y \in \mathfrak{M}$, the Fourier transform

$$
\Gamma_{X, Y}(s) \equiv \int_{\mathbb{R}}\left\langle\Omega \mid X \Delta^{i t} Y \Omega\right\rangle e^{i t s} \frac{\mathrm{d} t}{2 \pi}
$$

is well-defined in the sense of a tempered distribution in the variable $s$-in fact for $Y=X^{*}, \Gamma_{X, X^{*}}(s) \mathrm{d} s$ is a positive Radon measure on $\mathbb{R}$, see sec. 5.3 of $[17,18]$. By the Fourier inversion theorem, the operator $\Delta^{i t}$ is hence fully characterized provided we know $\Gamma_{X, Y}(s)$ for all $X, Y \in \mathfrak{M}$ and all $s$, i.e. as a distribution in $s$.

Using the KMS condition (8) after shifting the integration contour from the real axis $\mathbb{R}$ to the line $\mathbb{R}-i$ parallel to the real axis immediately gives

$$
\Gamma_{X, Y}(s)=e^{s} \Gamma_{Y, X}(-s) .
$$

On the other hand, for $X \in \mathfrak{M}, Y \in \mathfrak{M}^{\prime}$ or vice versa, we get

$$
\Gamma_{X, Y}(s)=\Gamma_{Y, X}(-s)
$$

using that the modular flow $\sigma^{t}(X)=\Delta^{i t} X \Delta^{-i t}$ preserves $\mathfrak{M}, \mathfrak{M}^{\prime}$.

We now want to describe how the extra structure of chiral conformal field theory can help to characterize $\Gamma_{X, Y}(s)$. The case we want to consider is the v. Neumann algebra $\mathfrak{M}=\mathfrak{A}(A)$, associated with a region $A$ consisting of $p$ open arcs. The vector under consideration is the vacuum, $|\Omega\rangle=\left|\Omega_{0}\right\rangle$. It seems that the information is most easily retrieved if instead of bounded operators $X, Y$, we work with point-like unbounded field operators as described in the previous section. We define for a generic primary field $\phi$ :

$$
\Gamma(s ; x, y) \equiv \int_{\mathbb{R}}\left\langle\Omega_{0}\left|\phi(x) \Delta^{i t} \phi(y)\right| \Omega_{0}\right\rangle e^{i t s} \frac{\mathrm{d} t}{2 \pi} .
$$


Here, $x, y \in \mathbb{S}$ are to be smeared with test functions on $A$ or $A^{\prime}$, and $\Delta \equiv \Delta_{A}$ is the modular operator in the vacuum state for the multi-interval/arc $A$. The quantity $\Gamma$ should be considered as analogous to (33).

Example 8: For one arc, $A=(a, b)$, the modular flow of a local primary field of dimension $h$ is given by the Hislop-Longo theorem $[8,9]$ as

$$
\Delta^{i t} \phi(x) \Delta^{-i t}=\left[g_{t}^{\prime}(x)\right]^{h} \phi\left(g_{t}(x)\right), \quad g_{t}(x)=\frac{a(b-x) e^{-2 \pi t}+b(x-a)}{(b-x) e^{-2 \pi t}+(x-a)} .
$$

Therefore $\Gamma$ can be found from (36) and (24), giving for $x, y \in(a, b)$

$$
\begin{aligned}
\Gamma(s, x, y)= & \frac{e^{-i s / 2-i \pi h}\left|\Gamma\left(h-\frac{i s}{2 \pi}\right)\right|^{2}}{(2 \pi)^{2} \Gamma(2 h)}\left(\frac{(b-a)^{2}}{(x-a)(x-b)(y-a)(y-b)}\right)^{h} . \\
& \cdot \exp \left(\frac{i s}{2 \pi} \ln \frac{(x-a)(y-b)}{(x-b)(y-a)}\right) .
\end{aligned}
$$

The example suggests that the behavior of $\Gamma(s, x, y)$ near a boundary point $q_{i}$ of a multi-interval could be $\left(x-q_{i}\right)^{-h}$. This is supported by the following lemma, formulated in the circle picture.

Lemma 1. Under the assumptions on the CFT given in the previous subsections:

1. If $f \in C_{0}^{\infty}(\mathbb{R})$ and $\Gamma(f ; x, y) \equiv \int \Gamma(s ; x, y) f(s) \mathrm{d} s$, then $\Gamma(f ; x, y)$ is smooth in $x, y \in \mathbb{S}$ away from the $2 p$ end-points of the $p$ intervals $I_{j}$. Moreover, for any $0<\varepsilon \leqslant \frac{1}{2}$,

$$
\begin{aligned}
& \left|\Gamma(f, x, y) \prod_{n=1}^{p}\left(x-a_{n}\right)^{h}\left(y-a_{n}\right)^{h}\left(x-b_{n}\right)^{h}\left(y-b_{n}\right)^{h}\right| \\
& \quad \leqslant C \varepsilon^{-2 h} \sup _{s}\left(e^{-\left(\frac{1}{2}-\varepsilon\right)|s|} e^{\sigma s / 2}|f(s)|\right)
\end{aligned}
$$

for some constant $C$ only depending on the end-points. Here, $\sigma=+1$ if both $x, y$ are in $A, \sigma=-1$ if $x, y$ are in $A^{\prime}=\mathbb{S} \backslash \bar{A}$.

2. We have the KMS condition

$$
\Gamma(s ; x, y)=e^{s} \Gamma(-s ; y, x)
$$

in the sense of distributions for $x, y \in A$. When $x \in A, y \in A^{\prime}$ (or vice versa), we have instead

$$
\Gamma(s ; x, y)=\Gamma(-s ; y, x) .
$$

Proof. 1) We will compare the quantity $\Gamma$ of an arbitrary multi-arc $A$ to that corresponding to a single arc. First we assume $x, y \in A$. Let $I$ be the largest $\operatorname{arc}$ contained in $A$ that is symmetric around $x$, and $J$ the largest interval contained in $A$ symmetric around $y$. If, for example, $c_{j}$ resp. $c_{k}$ are to the left of $x$ resp. $y$, then $I=\left(c_{j}, c_{j}^{-1} x^{2}\right)$ resp. $J=\left(c_{k}, c_{k}^{-1} y^{2}\right)$. We let $\Delta_{A}, \Delta_{J}, \Delta_{I}$ be the modular operators for the corresponding local algebras. Then we use the well-known operator inequality $\Delta_{I}^{\alpha} \geqslant \Delta_{A}^{\alpha}$ for $0 \leqslant \alpha \leqslant 1$. This follows from the fact that $\mathfrak{A}(A) \supset \mathfrak{A}(I)$, which is exploited as follows. Quite generally, let $\mathfrak{M}_{i}$ be two v. Neumann algebras on the same Hilbert space with common 
cyclic and separating vector $|\Omega\rangle$. We let $S_{i}$ be the Tomita operators for $\mathfrak{M}_{i}$ with polar decompositions $S_{i}=J_{i} \Delta_{i}^{1 / 2}$. Note that, if $\mathfrak{M}_{2} \subset \mathfrak{M}_{1}$, then $\mathcal{D}\left(S_{2}\right) \subset \mathcal{D}\left(S_{1}\right)$. The set $\mathcal{D}\left(S_{1}\right)$ is a Hilbert space called $\mathcal{H}_{1}$ with respect to the inner product (graph norm)

$$
(\Phi, \Psi)=\langle\Phi \mid \Psi\rangle+e^{u}\left\langle S_{1} \Psi \mid S_{1} \Phi\right\rangle=\left\langle\Phi \mid\left(1+e^{u} \Delta_{1}\right) \Psi\right\rangle .
$$

Letting $I: \mathcal{H}_{1} \rightarrow \mathcal{D}\left(S_{1}\right)$ be the identification map, one shows that $I^{-1} \mathcal{D}\left(S_{2}\right)$ is a closed subspace $\mathcal{H}_{2} \subset \mathcal{H}_{1}$ with associated orthogonal projection $P_{2}$. The operators $V_{j}=I^{-1}\left(1+e^{u} \Delta_{j}\right)^{-1 / 2}$ are isometries from $\mathcal{H}$ to $\mathcal{H}_{j}(j=1,2)$ and their adjoints are $V_{j}^{*}=\left(1+e^{u} \Delta_{j}\right)^{1 / 2} I P_{j}\left(\right.$ with $\left.P_{1}=1\right)$. There follow the relations

$$
\begin{aligned}
I P_{j} I^{*} & =I V_{j} V_{j}^{*} I^{*}=\left(1+e^{u} \Delta_{j}\right)^{-1}, \quad j=1,2 \\
I^{*} & =I^{-1}\left(1+e^{u} \Delta_{1}\right)^{-1}
\end{aligned}
$$

which can already be found in [24].

We multiply the first relation from the right with $X \in \mathfrak{M}_{2}$ and from the left with $Y^{*} \in \mathfrak{M}_{2}$ and take the expectation value in the state $|\Omega\rangle$. Then we use the second equation and obtain

$$
\begin{aligned}
& \left\langle\Omega\left|Y^{*}\left(1+e^{u} \Delta_{1}\right)^{-1} X\right| \Omega\right\rangle-\left\langle\Omega\left|Y^{*}\left(1+e^{u} \Delta_{2}\right)^{-1} X\right| \Omega\right\rangle \\
& \quad=\left\langle\left(1-P_{2}\right) I^{-1}\left(1+e^{u} \Delta_{1}\right)^{-1} Y \mid\left(1-P_{2}\right) I^{-1}\left(1+e^{u} \Delta_{1}\right)^{-1} X \Omega\right\rangle .
\end{aligned}
$$

The fact that the right side is manifestly non-negative for $X=Y$ implies $\left(1+e^{u} \Delta_{2}\right)^{-1} \leqslant$ $\left(1+e^{u} \Delta_{1}\right)^{-1}$, and that, combined with the operator identity

$$
\Delta_{1}^{\alpha}-\Delta_{2}^{\alpha}=\frac{\sin \pi \alpha}{\pi} \int_{-\infty}^{\infty} e^{-\alpha u}\left[\left(1+e^{u} \Delta_{2}\right)^{-1}-\left(1+e^{u} \Delta_{1}\right)^{-1}\right] \mathrm{d} u \leqslant 0
$$

for $0<\alpha<1$ gives the claim. Therefore $\Delta_{I}^{-\alpha / 2} \Delta_{A}^{\alpha / 2}\left(\Delta_{I}^{-\alpha / 2} \Delta_{A}^{\alpha / 2}\right)^{*}=\Delta_{I}^{-\alpha / 2} \Delta_{A}^{\alpha} \Delta_{I}^{-\alpha / 2}$ $\leqslant 1$ implying $\left\|\Delta_{A}^{\alpha / 2} \Delta_{I}^{-\alpha / 2}\right\| \leqslant 1$, and similarly for $J$. By the functional calculus, if $\mathrm{d} E(\lambda)$ is the spectral resolution of $\ln \Delta_{A}$ :

$$
\begin{aligned}
\Gamma(f, x, y) & =(2 \pi)^{-1} \int_{\mathbb{R}} e^{i t s} f(s) \int_{\mathbb{R}} e^{i \lambda t}\left\langle\Omega_{0}|\phi(x) \mathrm{d} E(\lambda) \phi(y)| \Omega_{0}\right\rangle \mathrm{d} \lambda \mathrm{d} s \\
& =\left\langle\Omega_{0}\left|\phi(x) f\left(-\ln \Delta_{A}\right) \phi(y)\right| \Omega_{0}\right\rangle,
\end{aligned}
$$

and then by the Cauchy-Schwarz inequality

$$
\begin{aligned}
|\Gamma(f, x, y)| & =\left|\left\langle\Omega_{0}\left|\phi(x) f\left(-\ln \Delta_{A}\right) \phi(y)\right| \Omega_{0}\right\rangle\right| \\
& \leqslant\left\|\Delta_{A}^{-\alpha} f\left(-\ln \Delta_{A}\right)\right\|\left\|\Delta_{A}^{\frac{\alpha}{2}} \Delta_{I}^{-\frac{\alpha}{2}}\right\|\left\|\Delta_{A}^{\frac{\alpha}{2}} \Delta_{J}^{-\frac{\alpha}{2}}\right\|\left\|\Delta_{I}^{\frac{\alpha}{2}} \phi(x) \Omega_{0}\right\| \\
& \left\|\Delta_{J}^{\frac{\alpha}{2}} \phi(y) \Omega_{0}\right\| \\
& \leqslant\left(\sup _{s} e^{\alpha s}|f(s)|\right)\left\|\Delta_{I}^{\frac{\alpha}{2}} \phi(x) \Omega_{0}\right\|\left\|\Delta_{J}^{\frac{\alpha}{2}} \phi(y) \Omega_{0}\right\| .
\end{aligned}
$$

Since $\phi(x)\left|\Omega_{0}\right\rangle$ can be analytically continued to a $\mathcal{H}_{0}$-valued holomorphic function inside the unit disk $\mathbb{D}^{+}$(by the mode expansion of $\phi$, see the previous subsection), the Hislop-Longo theorem applied to the modular flow $\sigma_{I}^{t}$ of $I$ can similarly be continued 
to imaginary flow time parameter $t=-i \alpha$ (see Eq. (37)). In combination with (24) we thereby obtain

$$
\begin{aligned}
\left\|\Delta_{I}^{\frac{\alpha}{2}} \phi(x) \Omega_{0}\right\|^{2} & =\left\langle\Omega_{0}\left|\phi(x)^{*} \Delta_{I}^{\alpha} \phi(x)\right| \Omega_{0}\right\rangle \\
& =\left\langle\Omega_{0}\left|\phi(x)^{*} \sigma_{I}^{-i \alpha}(\phi(x))\right| \Omega_{0}\right\rangle \\
& =x^{2 h}\left\langle\Omega_{0}\left|\phi(x) \sigma_{I}^{-i \alpha}(\phi(x))\right| \Omega_{0}\right\rangle \\
& =(2 \pi)^{-1}\left|\mathrm{~d} g_{-i \alpha}^{I}(x) / \mathrm{d} x\left(x-g_{-i \alpha}^{I}(x)\right)^{-2}\right|^{h} \\
& \lesssim(\sin \pi \alpha)^{-2 h}\left|x-c_{j}\right|^{-2 h},
\end{aligned}
$$

and similarly for $y$. Since this holds for any pair of end points,

$$
\begin{aligned}
& \left|\Gamma(f, x, y) \prod_{n=1}^{p}\left(x-a_{n}\right)^{h}\left(y-a_{n}\right)^{h}\left(x-b_{n}\right)^{h}\left(y-b_{n}\right)^{h}\right| \\
& \quad \lesssim(\sin \pi \alpha)^{-2 h} \sup _{s}\left(e^{\alpha s}|f(s)|\right)
\end{aligned}
$$

Now we split the testfunction $f=f_{+}+f_{-}$, where the testfunction $f_{-}$has support in $(-\infty, c]$, and the testfunction $f_{+}$has support in $[-c, \infty)$. Then, for the contribution from $f_{-}$, we choose $\alpha=1-\varepsilon$, whereas for the contribution from $f_{+}$, we choose $\alpha=\varepsilon$. As a consequence, we find that (39) holds.

This shows in particular boundedness in $x, y$ of $\Gamma(f, x, y)$ away from the endpoints. A similar estimation can be made for descendant fields, i.e. the derivatives of the fields $\phi(x), \phi(y)$, and this shows smoothness. This finishes the proof of 1) when $x, y \in A$.

To cover the other case, we note that the modular operator for $\mathfrak{A}(A)^{\prime} \supset \mathfrak{A}\left(A^{\prime}\right)$ is related to that of $\mathfrak{M}_{1}$ by $\Delta_{1}^{\prime}=\Delta_{1}^{-1}$. Then the other case of 1) follow by the same argument, namely, $x, y \in A^{\prime}$ we take $I^{\prime}$ be the largest interval contained in $A^{\prime}$ that is symmetric around $x$, and $J^{\prime}$ the largest interval contained in $A^{\prime}$ symmetric around $y$ and proceed in the same way as before.

2) Eq. (40) follows from the KMS condition (8) for bounded operators $X, Y \in$ $\pi_{0}(\mathfrak{A}(A))^{\prime \prime}$ because we are assuming about the local fields that for test-functions $f, g$ supported in $A$ there exist $X_{n}, Y_{n} \in \pi_{0}(\mathfrak{A}(A))^{\prime \prime}$ with the property that $\lim _{n} X_{n}\left|\Omega_{0}\right\rangle=$ $\phi(f)\left|\Omega_{0}\right\rangle$ and $\lim _{n} Y_{n}\left|\Omega_{0}\right\rangle=\phi(g)\left|\Omega_{0}\right\rangle$ in the strong topology. A similar remark applies to $(41)$.

We next make analytic continuations in the variables $x, y$. This gives us the following: For fixed $x \in A$, and fixed test-function $f(s)$, the function $y \mapsto \Gamma(f ; x, y)$ has an analytic extension to a holomorphic function of $y$ inside the unit disk $\mathbb{D}^{+}=\{z \in \mathbb{C} \mid$ $|z|<1\}$, by the mode expansion of $\phi$. Similarly, for fixed $y \in A$, the function $x \mapsto$ $\Gamma(s ; x, y)$ has an analytic extension to $x$ outside the unit disk $\mathbb{D}^{-}=\{z \in \mathbb{C}|| z \mid>1\}$. How to extend to $y \in \mathbb{D}^{-}$or $x \in \mathbb{D}^{+}$? The idea is that $\Gamma(f, y, x)$ is analytic in this domain, so we try to paste $\Gamma(s ; x, y)$ and $\Gamma(f, y, x)$ together across the boundary of the disk and hope that we get an analytic function that way. This will turn out to be the case on account of the KMS condition which relates the two quantities in precisely the right way.

To this end, we define the following auxiliary quantity for fixed $x \in A, s \in \mathbb{R}$ :

$$
K(y) \equiv \begin{cases}\Gamma(+s, x, y) & \text { if } y \in \mathbb{D}^{+} \\ \Gamma(-s, y, x) & \text { if } y \in \mathbb{D}^{-} .\end{cases}
$$


We similarly define, for fixed $y \in A, s \in \mathbb{R}$ :

$$
H(x) \equiv \begin{cases}\Gamma(+s, x, y) & \text { if } x \in \mathbb{D}^{-} \\ \Gamma(-s, y, x) & \text { if } x \in \mathbb{D}^{+}\end{cases}
$$

Note that $H$ implicitly also depends on the choice of $y, s$ and $K$ on the choice of $x, s$, but we suppress this since we are, for the moment, only interested in the dependence of $H$ on $x$ and of $K$ on $y$. Furthermore, note that $H$ and $K$ are, a priori defined only as holomorphic functions on the union $\mathbb{D}^{+} \cup \mathbb{D}^{-}=\mathbb{C} \backslash \mathbb{S}$, i.e. the complex plane minus the circle. On the circle, we define the boundary values from the inside resp. the outside of the disk $( \pm)$ :

$$
H^{ \pm}(x)=\lim _{z \rightarrow x, z \in \mathbb{D}^{ \pm}} H(z), \quad K^{ \pm}(y)=\lim _{z \rightarrow y, z \in \mathbb{D}^{ \pm}} K(z), \quad x, y \in \mathbb{S} .
$$

Then the "jump conditions" (40), (41) imply that

$$
H^{+}(x)=\left\{\begin{array}{ll}
H^{-}(x) & \text { if } x \in A^{\prime}, \\
e^{-s} H^{-}(x) & \text { if } x \in A,
\end{array} \quad K^{+}(y)= \begin{cases}K^{-}(y) & \text { if } y \in A^{\prime}, \\
e^{+s} K^{-}(x) & \text { if } y \in A .\end{cases}\right.
$$

Thus, both $H$ resp. $K$ are solutions to a Riemann-Hilbert-problem (across the contour $\mathbb{S}$ ). These problems are essentially completely understood, see e.g. [34,35]. The number and type of solutions depends in general on the specification of the behavior of $H$ resp. $K$ near the boundary points $\left\{q_{j}\right\}$ of the multi-arc $A=\cup_{j}\left(a_{j}, b_{j}\right)$ and at infinity, see e.g. [34] (para. 79, pp 230) or [35] (para. 42, pp 420). In the case at hand, this behavior is restricted by (39) and by the mode expansions of the fields.

A minor technical complication arises at this stage due to the fact that, as functions of $s$, both $H$ resp. $K$ are only defined in the distributional sense, and our bound (39) likewise also involves a test-function in $s$. This complication would make the direct application of the results in [34,35] somewhat cumbersome, so we give an explicit analysis of the implications imposed by the Riemann-Hilbert problem in the case at hand taking into account this complication.

First we define the shorthands

$$
\Pi_{a}(x)=\prod_{j=1}^{p}\left(x-a_{j}\right), \quad \Pi_{b}(x)=\prod_{j=1}^{p}\left(x-b_{j}\right),
$$

and

$$
Z_{ \pm}(x)=\frac{1}{2 \pi} \ln \left( \pm \prod_{j=1}^{p} \frac{\left(x-a_{j}\right)}{\left(x-b_{j}\right)}\right)
$$

Notice that $Z_{+}(x(1 \mp \varepsilon))=Z_{-}(x(1 \mp \varepsilon)) \mp i / 2$ when $\varepsilon \rightarrow 0^{+}$, and that $Z_{+}$has branch cuts on $A$, while $Z_{-}$has branch cuts on $A^{\prime}=\mathbb{S} \backslash \bar{A}$. Then we define

$$
\begin{aligned}
\widetilde{K}(y) & \equiv\left(\Pi_{a}(y) \Pi_{b}(y)\right)^{h} \int f(s) e^{i s\left(Z_{+}(x)-Z_{+}(y)\right)} K(y, s) \mathrm{d} s, \\
\widetilde{H}(x) & \equiv\left(\Pi_{a}(x) \Pi_{b}(x)\right)^{h} \int f(s) e^{i s\left(Z_{+}(x)-Z_{+}(y)\right)} H(x, s) \mathrm{d} s .
\end{aligned}
$$

We have: 
Lemma 2. $\widetilde{K}(y)$ is a polynomial in y of degree at most $2(p-1) h, \widetilde{H}(x)$ is a polynomial in $x$ of degree at most $2(p-1) h$.

Proof. We first consider $\widetilde{K}(y)$. The function $\ln \frac{\Pi_{a}(y)}{\Pi_{b}(y)}=2 \pi Z_{+}(y)$ jumps by $+2 \pi i$ as $y$ crosses $A$ from the inside of the unit disk to the outside. That jump compensates precisely the jump (40) implied by the KMS condition, so that $\widetilde{K}(y)$ is continuous across $A$. Similarly, (41) implies that $\widetilde{K}(y)$ is continuous across the complement $A^{\prime}$ as a function of $y$, too. Therefore, by the edge-of-the-wedge theorem, $\widetilde{K}$ is an analytic function of $y$ in the entire complex plane minus the boundary points of the intervals. Since $\widetilde{K}$ is a tempered distribution on $\mathbb{S}$ which away from the boundary points is the boundary value of an analytic function (from either inside or outside the unit disk), it follows that $\widetilde{K}$ cannot have any essential singularities at the boundary points $y \in\left\{q_{j}\right\}$. Indeed, at any given boundary point, say $a_{j}$, since we have a tempered distribution, there exists a natural number $N$, such that, if we multiply $\widetilde{K}(y)$ by $\left(y-a_{j}\right)^{N}$, we get a continuous function near $a_{j}$ in $y \in \mathbb{S}$. By the edge of the wedge theorem, this function, being a boundary value from both inside and outside the disk, must be holomorphic near $a_{j}$. Actually, by (39) we know the factor $\left(\Pi_{a}(y) \Pi_{b}(y)\right)^{h}$ cancels the potential blow up near $a_{j}$ when $y \in A$, and therefore we can actually choose $N=0$. Since this argument can be repeated for any other boundary point, we learn that the function $\widetilde{K}(y)$ is analytic in $y$ throughout the entire complex plane. A similar statement holds for $x$ and $y$ interchanged and with $\widetilde{K}$ and $\widetilde{H}$ interchanged.

We now establish a bound on the modulus of $\widetilde{K}(y)$ for $|y| \rightarrow \infty$. We learn from the mode expansions and properties of the fields that $y^{-2 p h+2 h} \widetilde{K}(y)$ remains bounded. Thus, we conclude that $|\widetilde{K}(y)| \lesssim|y|^{2 h p-2 h}$ throughout the entire complex plane for some new constant possibly depending on $x \in A$ and on the test-function $f$. Therefore, $\widetilde{K}(y)$ must for fixed $x \in A$ be a polynomial in $y$ of degree at most $2 p h-2 h$. We may repeat the same argument with the roles of $x$ and $y$ and of $\widetilde{K}$ and $\widetilde{H}$ reversed, and this finishes the proof.

The next lemma is a straightforward consequence of the preceding two lemmas.

Lemma 3. As a distribution on $(x, y) \in \mathbb{S} \times \mathbb{S}$ (and $s \in \mathbb{R})$, we have (for $Z_{-}$, see (56)):

$$
\begin{aligned}
& \Gamma(s, x, y) \\
& \quad=\left(\Pi_{a}(x) \Pi_{b}(x) \Pi_{a}(y) \Pi_{b}(y)\right)^{-h} \sum_{m, n=0}^{2(p-1) h} c_{m n}(s) q_{m}(x) \overline{q_{n}(y)} e^{i s\left[Z_{-}(y)-Z_{-}(x)\right]},
\end{aligned}
$$

where $\varepsilon^{2 h} e^{\left(\frac{1}{2}-\varepsilon\right)|s|-s / 2} c_{m n}(s) \in L^{1}(\mathbb{R}, \mathrm{d} s)$ for each $\frac{1}{2} \geqslant \varepsilon>0$, with uniformly bounded $L^{1}$-norm in $\varepsilon$, and where $q_{n}$ are polynomials of degree $n$.

Proof. We consider the distributional boundary values for $x \rightarrow A$ from within $\mathbb{D}^{-}$and for $y \rightarrow A$ from within $\mathbb{D}^{+}$, respectively in the following expression

$$
\widetilde{\Gamma}(s, x, y)=\lim _{x, y \rightarrow A}\left(\Pi_{a}(x) \Pi_{b}(x) \Pi_{a}(y) \Pi_{b}(y)\right)^{h} e^{i s\left[Z_{+}(x)-Z_{+}(y)\right]} \Gamma(s, x, y) .
$$

This boundary value prescription coincides with that for $\Gamma(s, x, y)$ and thus the right side is well-defined as a distribution in on $A \times A$ (after smearing in $s$ against a testfunction $f(s)$ ), by elementary results on products of distributions that are boundary values of 
analytic functions. By Lemma 2, $\widetilde{\Gamma}(f, x, y)$ is a polynomial both in $x$ and in $y$. The inequality (39) and the definition of $Z_{+}(56)$ gives the upper bound

$$
|\widetilde{\Gamma}(f, x, y)| \leqslant C \varepsilon^{-2 h} \sup _{s}\left(e^{-\left(\frac{1}{2}-\varepsilon\right)|s|-\frac{1}{2} s}|f(s)|\right)
$$

on this polynomial for all $x, y \in A$. Since the coefficients, $a_{m n}(f)$, of this polynomial can be reconstructed by interpolation from the values $\widetilde{\Gamma}\left(f, x_{\alpha}, y_{\beta}\right)$ for $2 h(p-1)$ interpolation points $x_{\alpha}$ and $2 h(p-1)$ interpolation points $y_{\beta}$ from $A$, this upper bound also holds for $a_{m n}(f)$. By the well-known duality between the Banach spaces $L^{1}(\mathbb{R})$ and $L^{\infty}(\mathbb{R})$ we can interpret this as saying that $a_{m n}(s) e^{\left(\frac{1}{2}-\varepsilon\right)|s|+\frac{1}{2} s}$ is a function in $L^{1}(\mathbb{R})$ with norm bounded from above by $C \varepsilon^{-2 h}$. Now set $c_{n m}(s)=a_{n m}(s) e^{s}$ and use the relationship $Z_{+}(x(1 \mp \varepsilon))=Z_{-}(x(1 \mp \varepsilon)) \mp i / 2$ when $\varepsilon \rightarrow 0^{+}$. Then the proposition follows after expressing $\Gamma$ in terms of $\widetilde{\Gamma}$.

If we wish, we can at this stage take an inverse Fourier transform of $G$ in $s$ to get a general expression for $\left\langle\Omega_{0}\left|\phi(x) \Delta^{i t} \phi(y)\right| \Omega_{0}\right\rangle$. We set

$$
\widehat{c}_{m n}(t)=\int_{\mathbb{R}} e^{-i t s} c_{m n}(s) \mathrm{d} s,
$$

and we may take the polynomials $q$ in (58) as monomials, again for simplicity of notation. Then we immediately get:

Proposition 1. 1) As a distribution in ( $x, y) \in A \times A$ (with boundary value prescription $(x, y) \in \mathbb{D}^{-} \times \mathbb{D}^{+} \rightarrow \mathbb{S} \times \mathbb{S}$ understood)

$$
\begin{aligned}
\left\langle\Omega_{0}\left|\phi(x) \Delta^{i t} \phi(y)\right| \Omega_{0}\right\rangle= & \left(\Pi_{a}(x) \Pi_{b}(x) \Pi_{a}(y) \Pi_{b}(y)\right)^{-h} . \\
& 2(p-1) h \\
& \cdot \sum_{m, n=0} \widehat{c}_{m n}(t+Z(x)-Z(y)) x^{m} y^{n},
\end{aligned}
$$

where $Z \equiv Z_{-}$is defined in (56), where $\widehat{c}_{m n}(t)$ is analytic in the strip $\{t \in \mathbb{C} \mid-1<$ $\Im(t)<0\}$. There, it satisfies a bound

$$
\left|\widehat{c}_{m n}(t)\right| \lesssim[\sin (\pi \Im t)]^{-2 h}
$$

and for real $t$ satisfies the property (in the distributional sense as a boundary value)

$$
\widehat{c}_{m n}(t-i)=\widehat{c}_{n m}(-t)=\overline{\widehat{c}_{m n}(t)} \text {. }
$$

2) We must have:

$$
\begin{aligned}
& \frac{(-1)^{h}}{2 \pi}\left(\frac{Q(x, y)}{2 \sinh \pi(Z(x)-Z(y)-i 0)}\right)^{2 h} \\
& =\sum_{m, n=0}^{2(p-1) h} \widehat{c}_{m n}(Z(x)-Z(y)-i 0) x^{m} y^{n}
\end{aligned}
$$

in the distributional sense (for $x, y \in A \subset \mathbb{S}$ ), where the bi-variate polynomial $Q$ is as in (66). 
Proof. 1) The formula (62) follows directly from Lemma 3. In particular, the claimed analyticity and bound (63) follow from the corresponding bounds on $c_{m n}(s)$. The formula (64) follows from the KMS-condition. 2) For $t=0$ we evidently have $\Delta^{i t}=1$. This condition gives a non-trivial constraint on the functions $\widehat{c}_{n m}$. Introduce the quantity $Z$ as in (56) and

$$
Q(x, y)=\frac{\prod_{j=1}^{p}\left(x-a_{j}\right)\left(y-b_{j}\right)-\prod_{j=1}^{p}\left(y-a_{j}\right)\left(x-b_{j}\right)}{x-y} .
$$

Note that $Q(x, y)$ is a polynomial in $x, y$ of degree $2(p-1)$ in each variable. We then get 2).

Remark 1. The domain of analyticity of $\widehat{c}_{m n}$ is large enough to permit us to take the limit $|y| \rightarrow \infty$ or $|x| \rightarrow 0$. The constraint then confirms that $\widehat{c}_{m n}(t)=0$ when $m, n>$ $2 h(p-1)$.

As we will see, in certain special cases eq. (65) and the properties given in proposition 1 suffice to determine $\widehat{c}_{m n}$ uniquely. For instance we will see in Sect. 4.2 that for a free fermion, the information we have obtained uniquely fixes the modular flow. For the $U(1)$-current, the proposition is however already less restrictive, although we are still able to get some results in Sect. 4.3. This is mainly because the polynomial $Q$ is of increasing degree and thus contains more free parameters for fields of higher dimension. Also for this reason, we will introduce in Sect. 5 another method.

4.2. Example: Modular flow of free Fermi field in vacuum (NS)-state. As an application of these general results, we find the action of the modular flow of a multi-arc $A$ for the net $\mathfrak{A}_{\text {Fermi }}$ in the vacuum state (Neveu-Schwarz sector), see example 7. Even though the free Fermi net is not local but graded local (the free Fermi field $\psi$ has $h=\frac{1}{2}$ ) we can easily adapt, in this simple case, our arguments leading to proposition 1 to fields obeying Fermi-statistics, i.e. fields of dimension $h \in \frac{1}{2} \mathbb{N}$. The main change appears in (41), where there is now a pre-factor -1 on the right side when $\phi=\psi$ obeys Fermi-statistics. This change propagates to eqs. (51) and (52), where there now appears a pre-factor -1 on the second line on the right sides in both equations. Following through this sign change one sees that proposition 1 still holds if we replace (64) by $\widehat{c}_{m n}(t-i)=-\widehat{c}_{n m}(-t)$.

To determine these functions, we may, in this simple case, test the relation (65) with $p$ points (and with $h=\frac{1}{2}$ ). We pick $\zeta, \eta \in \mathbb{R}$ not equal, and we let $x_{l}, y_{l} \in A \subset \mathbb{S}, k, l=$ $1, \ldots, p$ be the pre-images of $\zeta=Z\left(x_{l}\right) \neq \eta=Z\left(y_{k}\right)$, where $Z=Z_{-}$is the function defined by (56), and where $A$ is the union of $p$ open disjoint arcs in $\mathbb{S}$ as in (12). Testing the constraint (65) with these points we get for the free Fermi field $\psi$

$$
-\frac{i}{4 \pi} \frac{Q\left(x_{k}, y_{l}\right)}{\sinh \pi(\zeta-\eta-i 0)}=\sum_{m, n=0}^{p-1} \widehat{c}_{m n}(\zeta-\eta-i 0)\left(x_{k}\right)^{m}\left(y_{l}\right)^{n} .
$$

We note that $v_{m}\left(x_{k}\right)=\left(x_{k}\right)^{m}$ and $v_{n}\left(y_{l}\right)=\left(y_{l}\right)^{n}$ where $m, n=0, \ldots, p-1$ are $p \times p$ Vandermonde matrices whose determinants

$$
\operatorname{det}\left[v_{j}\left(x_{k}\right)\right]=\prod_{1 \leqslant i<j \leqslant p}\left(x_{i}-x_{j}\right) \neq 0
$$

do not vanish since all the points $x_{l}, l=1, \ldots, p$ are from disjoint intervals in $A$. Thus, the Vandermonde matrices in (67) may be inverted and therefore $\widehat{c}_{m n}(t)$ is uniquely 
determined. However, rather than finding the coefficients $Q(x, y)=\sum_{m, n=0}^{p-1} Q_{n m} x^{n} y^{m}$ from (66) and inserting the inverses of the Vandermondians directly, we may observe that one solution to the constraint (67) is of the form

$$
\widehat{c}_{n m}(t)=-\frac{i}{4 \pi} \frac{Q_{n m}}{\sinh \pi t}
$$

and this must hence be the unique solution. It is a good check that this solution is also consistent with the general properties of proposition 1 (for $h=\frac{1}{2}$ ). Substituting the solution into proposition 1 (for $h=\frac{1}{2}$ ) then gives:

Theorem 1. For the free massless real Fermifield on $\mathbb{S}$ and a multi-arc $A=\cup_{j=1}^{p}\left(a_{j}, b_{j}\right)$ $\subset \mathbb{S}$, the associated modular flow of the Neveu-Schwarz state is

$$
\left\langle\Omega_{0}\left|\psi(x) \Delta^{i t} \psi(y)\right| \Omega_{0}\right\rangle=\frac{1}{2 \pi i(x-y)} \frac{\Pi_{b}(x) \Pi_{a}(y)-\Pi_{a}(x) \Pi_{b}(y)}{e^{\pi t} \Pi_{b}(x) \Pi_{a}(y)-e^{-\pi t} \Pi_{a}(x) \Pi_{b}(y)},
$$

where $x, y \in A$, with the usual boundary value prescription ( $y$ approached from the within $\mathbb{D}^{+}$, $x$ approached from within $\mathbb{D}^{-}$) understood.

Since the action of the modular flow $\sigma^{t}$ is of second quantized form on the vacuum Hilbert space, it follows that modular flow is uniquely determined by (70). We now obtain the generator if the flow, thereby making contact with the original analysis due to [10] based on eigenfunctions of the Cauchy kernel.

First, we transform our result from the circle to the lightray via the Caley transformation $C: \mathbb{S} \backslash\{+1\} \rightarrow \mathbb{R}, C(x)=-i(x+1) /(x-1)$. The lightray fields are then related to the circle fields by $\psi_{\mathbb{S}}(x)=\sqrt{C^{\prime}(x)} \psi_{\mathbb{R}}(C(x))$. In terms of the lightray fields, eq. (70) is seen to retain its form, where the arcs of the circle $\left(a_{j}, b_{j}\right)$ become intervals $\left(C\left(a_{j}\right), C\left(b_{j}\right)\right)$ of the lightray. By abuse of notation, we can thus work with (70) and pretend that all quantities, such as $x, a_{j}, b_{j}, \psi, A$ (see (12)) refer to the lightray. Next, we go back from (70) to the Fourier transform (36) using (106). This gives us for $x, y \in A \subset \mathbb{R}$,

$$
\begin{aligned}
\langle 0|\psi(x)(\ln \Delta) \psi(y)| 0\rangle & =\int_{\mathbb{R}} s \Gamma(s, x, y) \mathrm{d} s \\
& =\frac{Q(x, y)}{4 \pi^{2}\left(\Pi_{a}(x) \Pi_{b}(x) \Pi_{a}(y) \Pi_{b}(y)\right)^{\frac{1}{2}}} \int_{\mathbb{R}} \frac{s e^{-i s Z_{-}(x)+i s Z_{-}(y)}}{1+e^{-s}} \mathrm{~d} s \\
& =\frac{1}{4 \pi^{2}} \sum_{k=1}^{p} \int_{\mathbb{R}} \frac{s}{1+e^{-s}} \overline{U_{s}^{k}(x)} U_{s}^{k}(y) \mathrm{d} s .
\end{aligned}
$$

In the last line we have substituted the functions $U_{s}^{k}(x)=\left(-\Pi_{a}(x) \Pi_{b}(x)\right)^{-\frac{1}{2}} q_{k}(x)$ $e^{i s Z_{-}(x)}$ [compare (62)] with the choice $q_{k}(x)=N_{k}^{-1} \prod_{i \neq k}\left(x-a_{i}\right)$ for the polynomials, where $N_{k}$ is the constant

$$
N_{k}^{2}=-\frac{\prod_{i \neq k}\left(a_{k}-a_{i}\right)}{\prod_{i=1}^{p}\left(a_{k}-b_{i}\right)}
$$

given in [10], and we have used the identity $\sum_{k=1}^{p} q_{k}(x) q_{k}(y)=Q(x, y)$ taken from [11] (eq. 2.55). For a function $f \in C_{0}^{\infty}(A, \mathbb{C})$ of compact support on our multi-interval $A$ (12), 
we next let $G_{A}$ be the restriction of the Cauchy kernel $G(x, y)=(1 / 2 \pi i)(x-y-i 0)^{-1}$ to $A$, defining an operator on $L^{2}(A)$ and we let

$$
k_{A}(x, y)=(2 \pi)^{-2} \sum_{k=1}^{p} \int_{\mathbb{R}} s \overline{U_{s}^{k}(x)} U_{s}^{k}(y) \mathrm{d} s,
$$

which will be identified as the modular hamiltonian on the 1-particle space momentarily. As shown by [11] (eq. 2.11), we have $G_{A} U_{k, s}=\left(1+e^{-s}\right)^{-1} U_{k, s}$, and the functions $U_{s}^{k}$ in fact give a spectral resolution of the operator $G_{A}$. We can consequently write, with $\psi(f)=\int_{\mathbb{R}} \psi(x) f(x) \mathrm{d} x, f, g \in C_{0}^{\infty}(A, \mathbb{C}):$

$$
\left\langle\Omega_{0}|\psi(f)(\ln \Delta) \psi(g)| \Omega_{0}\right\rangle=\left(G_{A} \bar{f}, k_{A} g\right)=\left(\bar{f}, G_{A} k_{A} g\right)
$$

with the usual $L^{2}$-inner product on the right side. The kernel $k_{A}(x, y)$ has been computed in [11] (eqs. 2.72, 2.76), with the result $\left(Z=Z_{-}\right)$

$$
\begin{aligned}
k_{A}(x, y)=-i & \left(\frac{1}{Z^{\prime}(x)} \delta^{\prime}(x-y)+\frac{1}{2}\left[\frac{1}{Z^{\prime}(x)}\right]^{\prime} \delta(x-y)\right. \\
& \left.-\frac{1}{x-y} \frac{1}{Z^{\prime}(x)} \sum_{j} \delta\left[x-y_{j}(Z(y))\right]\right)
\end{aligned}
$$

where the sum over $k$ is over all pre-images $y_{j}$ of $Z(y)$ not equal to $y$ itself. Below we will also see that $k_{A}=\ln \left(G_{A}^{-1}-1\right){ }^{4}$

In order to re-interpret this result on Fock space, it is convenient to give a slightly different, but fully equivalent description of the theory $\left\{\mathfrak{A}_{\mathrm{Fermi}}(I)\right\}$ on the lightray. The $n$-point functions on the lightray are of "quasi-free" form in the sense of [33]

$$
\left\langle\Omega_{0}\left|\psi\left(f_{1}\right) \cdots \psi\left(f_{n}\right)\right| \Omega_{0}\right\rangle= \begin{cases}\sum_{\sigma} \operatorname{sgn}(\sigma) \prod_{i=1}^{n / 2}\left(\bar{f}_{\sigma(i)}, G f_{\sigma(i+1)}\right) & n \text { even, } \\ 0 & \text { otherwise }\end{cases}
$$

where the sum is over all perfect matchings in the group of permutations on $n$ elements, and where $G$ is the operator defined by the Cauchy kernel. This operator is a projection which in momentum space corresponds to the multiplication with the characteristic function on $\mathbb{R}_{+}$, i.e. $\widehat{G f}(k)=1_{(0, \infty)}(k) \widehat{f}(k)$. As shown in [33], this leads to an alternative but equivalent description of $\mathcal{H}$ as the fermionic Fock-space $\mathcal{H}=\oplus_{n} \wedge^{n} \mathcal{K}$ with 1-particle space $\mathcal{K}=\left\{f \in L^{2}(\mathbb{R}) \mid \hat{f}(k)=0, \forall k \leqslant 0\right\}$ of square integrable functions $f(x)$ whose Fourier transform $\hat{f}(k)$ is non-zero only for $k \geqslant 0$. In terms of this Fock-space, the representation of the light ray fields can be written as

$$
\psi(f)=a^{*}(G f)+a(G \bar{f}),
$$

where $a^{*}(g), g \in \mathcal{K}$ are smeared creation operators defined as $a^{*}(g)|\Psi\rangle=|g \wedge \Psi\rangle$ on any $n$-particle state $|\Psi\rangle=\left|\Psi_{1} \wedge \cdots \wedge \Psi_{n}\right\rangle \in \wedge^{n} \mathcal{K} \subset \mathcal{H}$.

The 1-particle version of the Reeh-Schlieder theorem implies that it is consistent to introduce on the dense domain $\mathcal{D}\left(h_{A}\right)=\left\{G f \mid f \in C_{0}^{\infty}(A, \mathbb{C})\right\} \subset \mathcal{K}$ the "1-particle" modular Hamiltonian $h_{A}$ as

$$
h_{A}: \mathcal{D}\left(h_{A}\right) \rightarrow \mathcal{K}, \quad h_{A}(G f):=G k_{A} f,
$$

\footnotetext{
${ }^{4}$ In the literature [13], such formulas have previously been proven for finite-dimensional fermion algebras.
} 
and as a consequence of (89), we can then write the modular flow of $\mathfrak{A}_{\text {Fermi }}(A)$ for a multi-interval $A=\cup_{j=1}^{p} I_{j}$ in second quantized form as

$$
\Delta^{i t}=\bigoplus_{n=0}^{\infty} \bigwedge^{n} e^{i t h_{A}} .
$$

In view of (74) the final answer may also be (formally) rewritten as

$$
\left\langle\Omega_{0} \mid \psi(x)(\ln \Delta) \psi(y) \Omega_{0}\right\rangle=\left\langle\Omega_{0} \mid \psi(x)\left[H_{A}, \psi(y)\right] \Omega_{0}\right\rangle,
$$

where $H_{A}=\frac{1}{2} \int_{A \times A} k_{A}(x, y) \psi(x) \psi(y) \mathrm{d} x \mathrm{~d} y$ and $k_{A}$ the kernel of the operator on the right side of (75). Our result for the modular flow is thereby seen to be equivalent to the result for the modular flow found previously by [10]. Our arguments therefore in particular provide a rigorous proof of the result by [10]. For related results establishing (75) see $[32,36]$ who use different methods. They also give a (slightly) corrected way to write this equation in exponentiated form, see eq. 4.3 of [32].

4.3. Example: Modular flow of $U(1)$-current on $\mathbb{S}$. The conformal net $\mathfrak{A}_{U(1)}$ for the free $U$ (1) current algebra on the circle was defined in example 6. Via the Caley transform $C: \mathbb{S} \backslash\{+1\} \rightarrow \mathbb{R}, C(x)=-i(x+1) /(x-1)$, one obtains a corresponding net indexed by open intervals $I \subset \mathbb{R}$ of the real line (lightray) or a union thereof. The circle and lightray currents are related by $j_{\mathbb{S}}(x)=C^{\prime}(x) j_{\mathbb{R}}(C(x))$ and thus $j_{\mathbb{R}}(x)^{*}=j_{\mathbb{R}}(x)$. The corresponding lightray Weyl operators satisfy the same relations as on the circle. The two-point function on the lightray is

$$
\left\langle\Omega_{0}|j(x) j(y)| \Omega_{0}\right\rangle=-\frac{1}{2 \pi(x-y-i 0)^{2}},
$$

and thus takes the same form as on the circle (24) up to the precise form of the boundary value prescription.

We would next like to understand better the modular flow of the net $\mathfrak{A}_{U(1)}$ of the free $U$ (1) current algebra. In so far as proposition 1 is concerned, the discussion is actually identical for any bosonic field $\phi$ of dimension $d=1$. First we note that for local fields of conformal dimension $h=1,2,3, \ldots$ the method used for the free massless Fermi field to determine $\widehat{c}_{m n}$ is inapplicable since the analog of the Vandermonde matrices, $V_{l}^{n}=\left(x_{l}\right)^{n}, l=1, \ldots, p, n=1, \ldots, 2 h(p-1)$, that now appear in the analogue of (67) for general $h$ are no longer square matrices and hence not invertible as the sum over $n, m$ would now go up to $2 h(p-1)$ according to (65).

But we can obtain a weaker result for $h=1$ which will follow instantly from the following two lemmas. The first lemma is taken from [11].

Lemma 4. Let $x_{l} \in A \subset \mathbb{S}, l=1, \ldots, p$ ( $A$ the union of $p$ open disjoint arcs as in (12)) be the pre-images of $\zeta=Z_{-}\left(x_{l}\right)$ as in (56). Then

$$
\sum_{l=1}^{p} \frac{1}{2 \pi Z_{-}^{\prime}\left(x_{l}\right)} \frac{\left(x_{l}\right)^{j}}{\prod_{n=1}^{p}\left(x_{l}-a_{n}\right)\left(x_{l}-b_{n}\right)}=K_{j}
$$

for all natural numbers $j$ in the range $0 \leqslant j \leqslant 2 p-2$ and all $\zeta \in \mathbb{R}$, where

$$
K_{j}=\sum_{l=1}^{p} \frac{\left(a_{l}\right)^{j}}{\prod_{n=1}^{p}\left(a_{l}-b_{n}\right) \prod_{m \neq l}\left(a_{l}-a_{m}\right)}
$$




$$
=-\sum_{l=1}^{p} \frac{\left(b_{l}\right)^{j}}{\prod_{n=1}^{p}\left(b_{l}-a_{n}\right) \prod_{m \neq l}\left(b_{l}-b_{m}\right)}
$$

From this result, one gets:

Lemma 5. Let $x_{l}, y_{l} \in A \subset \mathbb{S}, k, l=1, \ldots, p$ be the pre-images of $\zeta=Z_{-}\left(x_{l}\right) \neq$ $\eta=Z_{-}\left(y_{k}\right)$ as in (56). Then

$$
\sum_{k, l=1}^{p} \frac{1}{Z_{-}^{\prime}\left(x_{k}\right)} \frac{1}{Z_{-}^{\prime}\left(y_{l}\right)} \frac{1}{(2 \pi)^{2}\left(x_{k}-y_{l}\right)^{2}}=\frac{p}{[2 \sinh \pi(\zeta-\eta)]^{2}} .
$$

Proof. Using the notation introduced in the previous proof (with $Z=Z_{-}$), we have:

$$
\begin{aligned}
& \sum_{k, l=1}^{p} \frac{1}{Z^{\prime}\left(x_{k}\right)} \frac{1}{Z^{\prime}\left(y_{l}\right)} \frac{1}{(2 \pi)^{2}\left(x_{k}-y_{l}\right)^{2}} \\
& \quad=\sum_{k, l=1}^{p} \frac{1}{Z^{\prime}\left(x_{k}\right)} \frac{1}{Z^{\prime}\left(y_{l}\right)} \frac{(2 \pi)^{-2} Q\left(x_{k}, y_{l}\right)^{2}}{\left(\Pi_{a}\left(x_{k}\right) \Pi_{b}\left(y_{l}\right)-\Pi_{a}\left(y_{l}\right) \Pi_{b}\left(x_{k}\right)\right)^{2}} \\
& \quad=\frac{(2 \pi)^{-2}}{\left(e^{\pi(\zeta-\eta)}-e^{\pi(\eta-\zeta))^{2}}\right.} \sum_{k, l=1}^{p} \frac{1}{Z^{\prime}\left(x_{k}\right) \Pi_{a}\left(x_{k}\right) \Pi_{b}\left(x_{k}\right)} \frac{1}{Z^{\prime}\left(y_{l}\right) \Pi_{a}\left(y_{l}\right) \Pi_{b}\left(y_{l}\right)} Q\left(x_{k}, y_{l}\right)^{2} \\
& \quad=\frac{1}{(2 \sinh \pi(\eta-\zeta))^{2}} \sum_{k, l=1}^{p} \frac{Q\left(a_{k}, a_{l}\right)^{2}}{\prod_{n=1}^{p}\left(a_{l}-b_{n}\right) \prod_{m \neq l}\left(a_{l}-a_{m}\right) \prod_{i=1}^{p}\left(a_{k}-b_{i}\right) \prod_{j \neq k}\left(a_{l}-a_{j}\right)}
\end{aligned}
$$

using the previous lemma in the last step. Now it follows from the definition of $Q$ (66) that

$$
Q\left(a_{k}, a_{l}\right)= \begin{cases}0 & \text { if } k \neq l, \\ -\left(\Pi_{a}^{\prime} \Pi_{b}-\Pi_{b}^{\prime} \Pi_{a}\right)\left(a_{k}\right) & \text { if } k=l,\end{cases}
$$

which is also equal to $-\delta_{k l} \prod_{j \neq l}\left(a_{l}-a_{j}\right) \prod_{i=1}^{p}\left(a_{l}-b_{i}\right)$. Inserting this identity into (85) completes the proof.

Now let $\phi$ be a bosonic field of dimension $d=1$. For fixed $\zeta$, consider the pre-images $x_{l} \in A, l=1, \ldots, p$ of $\zeta=Z_{-}\left(x_{l}\right)$ inside the $p$ open disjoint arcs (12). We can view the $x_{l}=x_{l}(\zeta)$ as functions of $\zeta$ and form the operator-valued distribution on $\mathbb{R}$ given by

$$
\widetilde{\phi}(\zeta)=\frac{1}{2 \pi} \sum_{l=1}^{p} x_{l}^{\prime}(\zeta) \phi\left(x_{l}(\zeta)\right)
$$

formally corresponding to the "transformation law" of a primary field of dimension 1. Our first result on the modular flow is

Theorem 2. We have for any dimension 1 fields $\phi$ (e.g. the U(1)-current)

$$
\left\langle\Omega_{0}\left|\widetilde{\phi}(\zeta) \Delta^{i t} \widetilde{\phi}(\eta)\right| \Omega_{0}\right\rangle=\frac{p}{[2 \sinh \pi(t+\zeta-\eta-i 0)]^{2}}
$$

in the sense of distributions in $\eta, \zeta \in \mathbb{R}$. 
Proof. First we apply eq. (24) to $\phi$ and we set $x=x_{k}, y=y_{l}$ and sum over $k, l=$ $1, \ldots, p$. Then we get from the previous lemma:

$$
\left\langle\Omega_{0}|\tilde{\phi}(\zeta) \widetilde{\phi}(\eta)| \Omega_{0}\right\rangle=\frac{p}{[2 \sinh \pi(\zeta-\eta-i 0)]^{2}}
$$

Next we set $x=x_{k}, y=y_{l}$ in proposition 1 and sum over $k, l=1, \ldots, p$. Then we get using the notation introduced in the previous proofs

$$
\begin{aligned}
& \left\langle\Omega_{0}\left|\tilde{\phi}(\zeta) \Delta^{i t} \widetilde{\phi}(\eta)\right| \Omega_{0}\right\rangle \\
& =(2 \pi)^{-2} \sum_{k, l=1}^{p} \sum_{m, n=0}^{2(p-1)} \frac{\widehat{c}_{m n}(t+\zeta-\eta-i 0)\left(x_{k}\right)^{m}\left(y_{l}\right)^{n}}{Z^{\prime}\left(x_{k}\right) Z^{\prime}\left(y_{l}\right) \Pi_{a}\left(x_{k}\right) \Pi_{a}\left(y_{l}\right) \Pi_{b}\left(x_{k}\right) \Pi_{b}\left(x_{l}\right)} \\
& =\sum_{m, n=0}^{2(p-1)} \widehat{c}_{m n}(t+\zeta-\eta-i 0) K_{m} K_{n}
\end{aligned}
$$

applying Lemma 4 in the last step. Now let $f(t)=\sum_{m, n=0}^{2(p-1)} \widehat{c}_{m n}(t) K_{m} K_{n}$. Comparing (90) with (89), we conclude that $f(t-i 0)=p[2 \sinh (\pi t-i 0)]^{-2}$ for real $t$, and hence for all $t$ in the strip $0>\Im t>-1$ by the edge-of-the-wedge theorem, completing the proof.

Remark 2. As is well-known, the $U(1)$ current can be represented on the Fock space of two independent free real Fermion fields $\psi_{1}, \psi_{2}$ by $j(x)=i: \psi_{1} \psi_{2}:(x)$. In operator algebraic terms, the $U(1)$-net is a subnet of two copies of the free Fermi net, see [27]. If one could show that there was a unit norm vacuum preserving conditional expectation value from the Fermi algebra for region $A$ to the current algebra of $A$ (as follows from the work by [27] when $A$ is an interval by Haag duality), then the modular flow on the current algebra $\mathfrak{A}_{U(1)}(A)$ would be that induced by the flow for the Fermi net (Theorem 1), by Takesaki's theorem, see e.g. sec. 5 of [37]. At present, however, we do not know that such a conditional expectation exists for multi-component regions $A$.

As a test, Theorem 1, eq. (70), can then be applied to $\widetilde{j}(\zeta)$ defined as in (87). Alternatively, we my compute the left side of (70) using the modular flow of the free Fermi field(s) given explicitly in $[10,32]$. One sees after a computation that either results are consistent with Theorem 2. In the next section, we will again discuss the modular Hamiltonian for the $U(1)$-current from a different perspective.

\section{Thermal States}

It is possible to analyse the modular flow of a thermal state in a similar manner as for the vacuum state. However, we find it useful to use a variation of the method described in the previous section which, in essence, corresponds to replacing the matrix elements of the function $\Delta^{i t}$ with functions closely related to the resolvent, $(\Delta-\lambda)^{-1}$. Such matrix elements will have certain analogous jump properties as the functions $K, H$ introduced above, but their exact form depends on the statistics (i.e. conformal dimension $h$ ) of the field $\phi(x)$. The cases of fermionic ${ }^{5}$ and bosonic fields are treated separately in Sects. 5.1 and 5.3 , respectively.

\footnotetext{
5 In the case of fermionic fields, we have graded locality as in the example of the free Fermi field.
} 
We will use the parametrization $x=e^{2 \pi i u}$ of the circle. Under this map $A$ consists of intervals $\cup_{i=1}^{p}\left(a_{i}, b_{i}\right) \subset(0,1)$. $A^{\prime}$ is as before the interior of the complement. We define, by a slight abuse of notations,

$$
\phi(u) \equiv e^{2 \pi i h u} \phi\left(e^{2 \pi i u}\right) .
$$

A Gibbs state is given by the usual formula

$$
\omega_{\beta}(X)=\frac{\operatorname{Tr}\left(X e^{-\beta L_{0}}\right)}{\operatorname{Tr}\left(e^{-\beta L_{0}}\right)}, \quad \Re(\beta)>0, \quad X \in \mathfrak{A}(A),
$$

where the trace is taken in the vacuum (i.e. the defining) representation $\left(\pi_{0}, \mathcal{H}_{0}\right)$ of the net. We shall mostly take $\beta$ to be real and positive, and occasionally use $\tau=$ $i \beta / 2 \pi$, which is the periodicity of the correlation functions in imaginary direction in the coordinate $u$. The general case can be obtained usually by analytic continuation in the end. Then it follows immediately that $\omega_{\beta}$ is a $\beta$-KMS-state on $\mathfrak{A}=\mathfrak{B}\left(\mathcal{H}_{0}\right)$ relative to the 1-parameter automorphism group of rotations of the circle, i.e. translations in $u$. By the Reeh-Schlieder theorem, the GNS-vector $\left|\Omega_{\beta}\right\rangle$ corresponding to $\omega_{\beta}$ is (cyclic and) separating for $\mathfrak{A}(A)=\vee_{i=1}^{p} \mathfrak{A}\left(I_{i}\right)$, and we can define a corresponding modular operator $\Delta \equiv \Delta_{\beta, A}$ as in the vacuum situation.

5.1. Fermionic fields. We begin by introducing a variant of the construction in the previous section involving resolvents. For $|\Omega\rangle \equiv\left|\Omega_{\beta}\right\rangle$ and $\Delta \equiv \Delta_{\beta, A}$, we set ${ }^{6}$

$$
F(\xi, u, v)=\left\{\begin{array}{lc}
\left\langle\Omega \mid \phi(u)\left[\left(\Delta^{-1}+1\right)^{-1}+\xi-\frac{1}{2}\right]^{-1} \phi(v) \Omega\right\rangle & \text { if }-\beta / 2 \pi<\Im(u)<0, \\
-\left\langle\Omega \mid \phi(v)\left[(\Delta+1)^{-1}+\xi-\frac{1}{2}\right]^{-1} \phi(u) \Omega\right\rangle & \text { if } \beta / 2 \pi>\Im(u)>0 .
\end{array}\right.
$$

The resolvents in these expressions are well defined if $\xi \in\left(\frac{1}{2}, \infty\right) \cup\left(-\frac{1}{2},-\infty\right)$ in view of $\Delta>0$, and the analytic continuations in $u$ are justified by the KMS property for the Gibbs state, because $\phi(u)|\Omega\rangle$ is a vector-valued holomorphic function on the strip $\{u \in \mathbb{C} \mid 0<\Im(u)<\beta / 2 \pi\}$. $\phi$ is assumed to be a hermitian field of conformal dimension $h$ satisfying our Assumptions 1. The relation to the modular Hamiltonian follows from the formula

$$
-\ln \Delta=\int_{\frac{1}{2}}^{\infty} \mathrm{d} \xi\left(\left[\left(\Delta^{-1}+1\right)^{-1}+\xi-\frac{1}{2}\right]^{-1}+\left[\left(\Delta^{-1}+1\right)^{-1}-\xi-\frac{1}{2}\right]^{-1}\right),
$$

which trivially holds for positive real numbers $\Delta$ and for positive self adjoint operators in view of the spectral theorem. This immediately gives

$$
\langle\Omega \mid \phi(u)(\ln \Delta) \phi(v) \Omega\rangle=-\int_{\frac{1}{2}}^{\infty} \mathrm{d} \xi(F(\xi, u, v)+F(-\xi, u, v)),
$$

where $u$ has a small negative imaginary part. The holomorphic functional calculus also implies more general formulas such as

$$
\langle\Omega \mid \phi(u) h(\Delta) \phi(v) \Omega\rangle
$$

\footnotetext{
${ }^{6}$ Here and in the following, we write $\phi(u)$ for $\pi_{\omega}(\phi(u))$, where $\pi_{\omega}$ is the GNS representation of $\omega \equiv \omega_{\beta}$.
} 


$$
=\frac{1}{2 \pi i} \int_{-\infty}^{\infty} \mathrm{d} \xi h\left(\frac{1 / 2+\xi}{1 / 2-\xi}\right)(F(\xi+1+i 0, u, v)-F(\xi+1-i 0, u, v)),
$$

for suitable holomorphic $h$.

In any case, we should try to find $F$. Our first lemma is the crucial tool expressing the analyticity/jump properties across the real $u$-axis.

Lemma 6. Let $v \in A$ be fixed. If $u \in A^{\prime}$, then

$$
F(\xi, u-i 0, v)=F(\xi, u+i 0, v) .
$$

If $u \in A$, then

$$
-\left(\xi+\frac{1}{2}\right) F(\xi, u+i 0, v)+\left(\xi-\frac{1}{2}\right) F(\xi, u-i 0, v)=\langle\Omega \mid\{\phi(u), \phi(v)\} \Omega\rangle,
$$

in the sense of distributions. Here $\{\phi(u), \phi(v)\}=\phi(u) \phi(v)+\phi(v) \phi(u)$ is the anticommutator.

Proof. We take $\lambda=\frac{1}{2}-\xi$ and consider the elementary formula

$$
\frac{1}{\lambda} \frac{\Delta^{-1}+1}{\Delta^{-1}+1-1 / \lambda}=\frac{1}{(\lambda-1)+(\Delta+1)^{-1}} .
$$

Using this formula, and $u, v \in A$, the definition of $F$ gives

$$
\begin{aligned}
F & (\xi, u+i 0, v)=-\left\langle\Omega \mid \phi(v)\left[(\Delta+1)^{-1}+(\lambda-1)\right]^{-1} \phi(u) \Omega\right\rangle \\
& =-\frac{1}{\lambda}\left\langle\Omega \mid \phi(v)\left(\Delta^{-1}+1\right)\left(\Delta^{-1}+1-1 / \lambda\right)^{-1} \phi(u) \Omega\right\rangle \\
& =-\frac{1}{\lambda}\langle\Omega \mid \phi(v) \phi(u) \Omega\rangle-\frac{1}{\lambda^{2}}\left\langle\Omega \mid \phi(v)\left(\Delta^{-1}+1-1 / \lambda\right)^{-1} \phi(u) \Omega\right\rangle .
\end{aligned}
$$

On the other hand, using the formula for $\Delta \rightarrow \Delta^{-1}$ and the definition of $F$ gives also

$$
\begin{aligned}
& F(\xi, u-i 0, v)=\left\langle\Omega \mid \phi(u)\left[\left(\Delta^{-1}+1\right)^{-1}+(\lambda-1)\right]^{-1} \phi(v) \Omega\right\rangle \\
&= \frac{1}{\lambda-1}\langle\Omega \mid \phi(u) \phi(v) \Omega\rangle-\frac{1}{\lambda-1}\langle\Omega| \phi(u)\left(\Delta^{-1}+1\right)^{-1} \\
& {\left.\left[\left(\Delta^{-1}+1\right)^{-1}+(\lambda-1)\right]^{-1} \phi(v) \Omega\right\rangle } \\
&= \frac{1}{\lambda-1}\langle\Omega \mid \phi(u) \phi(v) \Omega\rangle-\frac{1}{\lambda(\lambda-1)}\langle\Omega| \phi(u)\left(\Delta^{-1}+1\right)^{-1}(\Delta+1) \\
& {\left.[\Delta+1-1 / \lambda]^{-1} \phi(v) \Omega\right\rangle } \\
&= \frac{1}{\lambda-1}\langle\Omega \mid \phi(u) \phi(v) \Omega\rangle-\frac{1}{\lambda(\lambda-1)}\langle\Omega| \phi(v) \Delta(\Delta+1)^{-1}\left(\Delta^{-1}+1\right) \\
& {\left.[\Delta+1-1 / \lambda]^{-1} \phi(u) \Omega\right\rangle } \\
&= \frac{1}{\lambda-1}\langle\Omega \mid \phi(u) \phi(v) \Omega\rangle-\frac{1}{\lambda(\lambda-1)}\left\langle\Omega \mid \phi(v)\left(\Delta^{-1}+1-1 / \lambda\right)^{-1} \phi(u) \Omega\right\rangle .
\end{aligned}
$$


Here, we used the relations $\phi(u)|\Omega\rangle=\phi(u)^{*}|\Omega\rangle=S \phi(u)|\Omega\rangle=J \Delta^{\frac{1}{2}} \phi(u)|\Omega\rangle$, the anti-unitary property of $J$, and the property $J \Delta^{-1} J=\Delta$, which follow from TomitaTakesaki theory. Adding up these relations:

$$
(\lambda-1) F(\xi, u-i 0, v)-\lambda F(\xi, u+i 0, v)=\langle\Omega \mid\{\phi(u), \phi(v)\} \Omega\rangle,
$$

which is equivalent to the statement of the lemma when $u, v \in A$.

In the other case, when $u \in A^{\prime}, v \in A$, we use the formula (117). On this formula, we act from the left with $\phi(v)$ and from the left with $\phi(u)$ and take the expectation value in $\mid \Omega$. Then we obtain a formula for $F$. Since $u \in A^{\prime}, v \in A$, we have $\left[\Delta^{-i t} \phi(v) \Delta^{i t}, \phi(u)\right]=0$ by Tomita-Takesaki theory, and using this formula to commute the operators inside the expectation values, we get the claim of the lemma when $u \in A^{\prime}, v \in A$.

As in the previous section, the lemma shows that $F(\xi, u, v)$ defines a function of $u$ for fixed $v \in A$ that is analytic in the cut strip $\{u \in \mathbb{C}|| \Im(u) \mid<\beta / 2 \pi, u \notin A\}$. Furthermore, by the KMS condition, it can be checked that $H(s, u-i(\beta-0), v)=$ $-H(s, u+i 0, v)$, which is also $=-H(s, u+1+i 0, v)$ by construction. Thus, $F(\xi, u, v)$ has the same periodicity as the 2-point function $\langle\Omega \mid \phi(u) \phi(v) \Omega\rangle$, that is

$$
F(\xi, u+1, v)=F(\xi, u+\tau, v)=-F(\xi, u, v) .
$$

This allows us to define $F$ as a function of $u$ on the entire complex plane cut by $A+\mathbb{Z}+\tau \mathbb{Z}$. The limits from below the real axis define hermitian distributional kernels $F(\xi, u-i 0, v)$ that are of positive/negative type for $\xi>\frac{1}{2}$ resp. $\xi<-\frac{1}{2}$, which follows from $\Delta>0$; similarly for the limit from above the real axis. Similar statements hold when fixing $u \in A$ and viewing $F(\xi, u, v)$ as a function of $v$.

As in the vacuum case studied in Sect. 4.1, we would next like to have a result like (39) of Lemma 1 about the potential singularities of $F$ at the end points of the intervals. Unfortunately, the proof strategy of Lemma 1 does not hold in the present case since we have no analogue of the Hislop-Longo theorem for thermal states.

Instead, we will prove first a result comparing the modular operator of a thermal state $\omega_{\beta}$ for the full algebra $\mathfrak{A}=\mathfrak{B}\left(\mathcal{H}_{0}\right)$ to the modular operator for the partial algebra $\mathfrak{A}(A)$. The point is that the former corresponds to rotations of the circle (i.e. translations of the coordinate $u$ ) and is thus known. The idea is more precisely to apply eq. (45) to the case $\mathfrak{M}_{1}=\mathfrak{A}, \mathfrak{M}_{2}=\mathfrak{A}(A)$ (viewed as operator algebras on the GNS-Hilbert space $\mathcal{H}_{\beta} \equiv \mathcal{H}$ of the thermal state $\left.\omega_{\beta} \equiv \omega\right)$, so we can define the modular operators $\Delta_{i}$ of $\mathfrak{M}_{i}$ on $\mathcal{H}$ with respect to the cyclic and separating vector $|\Omega\rangle \equiv\left|\Omega_{\omega}\right\rangle$.

Lemma 7. Let $s \in \mathbb{R}$, let $u, v \in A=\cup_{i=1}^{p}\left(a_{i}, b_{i}\right)$. Then

$$
\begin{aligned}
& \left|\left\langle\Omega \mid \phi(u)\left(1+e^{s} \Delta_{1}\right)^{-1} \phi(v) \Omega\right\rangle-\left\langle\Omega \mid \phi(u)\left(1+e^{s} \Delta_{2}\right)^{-1} \phi(v) \Omega\right\rangle\right| \\
& \quad \lesssim \sum_{q_{j}}\left|u-q_{j}\right|^{-h}\left|v-q_{j}\right|^{-h},
\end{aligned}
$$

with implicit constant depending on s and the endpoints $\left\{q_{j}\right\}$ of the intervals.

Proof. Let $X, Y \in \mathfrak{M}_{2}$. From (45), we get with the notations introduced around (42)

$$
\begin{aligned}
& \left\langle\Omega\left|Y^{*}\left(1+e^{s} \Delta_{1}\right)^{-1} X\right| \Omega\right\rangle-\left\langle\Omega\left|Y^{*}\left(1+e^{s} \Delta_{2}\right)^{-1} X\right| \Omega\right\rangle \\
& \quad=\left\langle\left(1-P_{2}\right) I^{-1}\left(1+e^{s} \Delta_{1}\right)^{-1} Y \Omega \mid\left(1-P_{2}\right) I^{-1}\left(1+e^{s} \Delta_{1}\right)^{-1} X \Omega\right\rangle .
\end{aligned}
$$


For $y>0$, we can write

$$
\frac{1}{1+y}=\frac{i}{2} \int_{\mathbb{R}} \frac{y^{i t}}{\sinh [\pi(t+i 0)]} \mathrm{d} t .
$$

Therefore, by the spectral calculus applied to $y=e^{s} \Delta_{1}$,

$$
\left(1-P_{2}\right) I^{-1}\left(1+e^{s} \Delta_{1}\right)^{-1} X|\Omega\rangle=\frac{i}{2}\left(1-P_{2}\right) I^{-1} \int_{\mathbb{R}} \mathrm{d} t \frac{e^{i s t} \Delta_{1}^{i t}}{\sinh [\pi(t+i 0)]} X|\Omega\rangle .
$$

The key idea is now the following. Suppose that, for $|t|<t_{0}$ and some $t_{0}>0$, we knew that $\sigma_{1}^{t}(X)$ is in $\mathfrak{M}_{2}$, so $\Delta_{1}^{i t} X|\Omega\rangle=\sigma_{1}^{t}(X)|\Omega\rangle$ is in the domain $\mathcal{D}\left(S_{2}\right)$, so $I^{-1} \Delta_{1}^{i t} X|\Omega\rangle$ is in $\mathcal{H}_{2}$, so $\left(1-P_{2}\right) I^{-1} \Delta_{1}^{i t} X|\Omega\rangle=0$. Then we can effectively restrict the range in the integral to $|t| \geqslant t_{0}$ and drop the $i 0$-prescription, and this is the moral reason for the existence of our bound.

An even better estimate is obtained if instead we choose a, say even, real-valued smooth function $h(t) \geqslant 0$ such that $h(t)=0$ for $|t|<\frac{1}{2}$ and $h(t)=1$ for $|t| \geqslant 1$, say, and write

$$
\left(1-P_{2}\right) I^{-1}\left(1+e^{s} \Delta_{1}\right)^{-1} X|\Omega\rangle=\frac{i}{2}\left(1-P_{2}\right) I^{-1} \int_{\mathbb{R}} \mathrm{d} t \frac{e^{i s t} \Delta_{1}^{i t}}{\sinh (\pi t)} h\left(\frac{t}{t_{0}}\right) X|\Omega\rangle .
$$

Now we take a test function $f$ compactly supported inside $A$ and we denote the distance of the support of $f$ to the boundary of $A$ by $\delta=\operatorname{dist}(\partial A, \operatorname{supp}(f))$; of course $\delta>0$. Furthermore, we let $\left\{X_{n}\right\}$ be a sequence in $\mathfrak{M}_{2}$ converging strongly to $\phi(f)$. Such a sequence exists since we assume that the local fields are affiliated. Then (108) also holds for $X=\phi(f)$. We next wish to use the mode expansion (21) inside (108). Because $\omega \equiv \omega_{\beta}$ is a $\beta$-KMS state on $\mathfrak{M}_{1}$ with respect to the automorphic actions of rotations $\alpha_{t}=\alpha_{g_{t}}, g_{t}(z)=e^{-i \beta t} z$, the modular flow $\Delta_{1}^{i t}$ corresponds to rotations in the sense that $\Delta_{1}^{i t} \phi(z) \Delta_{1}^{-i t}=e^{-i h t \beta} \phi\left(e^{-i \beta t} z\right)$ for $z \in \mathbb{S}$. Then it is clear that $\Delta_{1}^{i t} \phi(f) \Delta_{1}^{-i t}$ will remain affiliated with $\mathfrak{M}_{2}$ as long as $|t|<\delta / \beta$, so (108) holds with $X=\phi(f)$ and $t_{0}=\delta / \beta$.

In terms of modes

$$
\Delta_{1}^{i t} \phi_{n} \Delta_{1}^{-i t}=e^{i \beta(n+h) t} \phi_{n}
$$

In particular, since the modular flow preserves the state, we must have $\left\langle\phi_{m} \Omega \mid \phi_{n} \Omega\right\rangle=$ $\delta_{n, m}\left\|\phi_{n} \Omega\right\|^{2}=\delta_{n, m} \omega\left(\phi_{n}^{*} \phi_{n}\right)$. From (iv) of Assumption 1 it follows that $\phi_{n}^{*} \phi_{n} \lesssim(1+$ $|n|)^{2 h-1}\left(1+L_{0}\right)^{2 k}$ for some $k \geqslant 0$, and therefore we must have

$$
\left\|\phi_{n} \Omega\right\|^{2} \lesssim(1+|n|)^{2 h-1} \omega\left[\left(1+L_{0}\right)^{2 k}\right]
$$

for all $n$. Since $\omega \equiv \omega_{\beta}$ is a Gibbs state, we conclude

$$
\left\|\phi_{n} \Omega\right\|^{2} \lesssim(1+|n|)^{2 h-1} \frac{\operatorname{Tr}\left[\left(1+L_{0}\right)^{2 k} e^{-\beta L_{0}}\right]}{\operatorname{Tr}\left(e^{-\beta L_{0}}\right)} \lesssim(1+|n|)^{2 h-1}
$$


Using the definition of $I$ as well as $1-P_{2} \leqslant 1$, we get for any complex constants $c_{n}$,

$$
\begin{aligned}
\left\|\sum_{n \in \mathbb{Z}} c_{n}\left(1-P_{2}\right) I^{-1} \phi_{n} \Omega\right\|^{2} & \leqslant \sum_{n, m \in \mathbb{Z}} \bar{c}_{n} c_{m}\left\langle I^{-1} \phi_{m} \Omega \mid I^{-1} \phi_{n} \Omega\right\rangle \\
& =\sum_{n, m \in \mathbb{Z}} \bar{c}_{n} c_{m}\left\langle\phi_{m} \Omega \mid\left(1+e^{s} \Delta_{1}\right) \phi_{n} \Omega\right\rangle \\
& =\sum_{n \in \mathbb{Z}}\left|c_{n}\right|^{2}\left(\left\|\phi_{n} \Omega\right\|^{2}+e^{s}\left\|\phi_{n}^{*} \Omega\right\|^{2}\right) \\
& =\sum_{n \in \mathbb{Z}}\left|c_{n}\right|^{2}\left(\left\|\phi_{n} \Omega\right\|^{2}+e^{s}\left\|\phi_{-n} \Omega\right\|^{2}\right) \\
& \leqslant\left(1+e^{s}\right) \sum_{n}\left|c_{n}\right|^{2} O\left(|n|^{2 h-1}\right) .
\end{aligned}
$$

Taking the norm squared of (108) then gives:

$$
\begin{aligned}
& \left\|\left(1-P_{2}\right) I^{-1}\left(1+e^{s} \Delta_{1}\right)^{-1} \phi(f) \Omega\right\|^{2} \\
& =\frac{1}{4}\left\|\sum_{n \in \mathbb{Z}}\left(\int_{\mathbb{R}} \frac{e^{i s t+i \beta(n+h) t}}{\sinh (\pi t)} h\left(\frac{\beta t}{\delta}\right) \mathrm{d} t\right) f_{n}\left(1-P_{2}\right) I^{-1} \phi_{n} \Omega\right\|^{2} \\
& \quad \leqslant\left(1+e^{s}\right) \sum_{n \in \mathbb{Z}}\left|\int_{\mathbb{R}} \frac{e^{i s t+i \beta(n+h) t}}{\sinh (\pi t)} h\left(\frac{\beta t}{\delta}\right) \mathrm{d} t\right|^{2}\left|f_{n}\right|^{2} O\left(|n|^{2 h-1}\right) \\
& \equiv\left(1+e^{s}\right) \sum_{n \in \mathbb{Z}} \varphi_{\delta / \beta}(s+\beta(n+h))\left|f_{n}\right|^{2} O\left(|n|^{2 h-1}\right),
\end{aligned}
$$

where $f_{n}=\int_{0}^{1} e^{2 \pi i n u} f(u) \mathrm{d} u$ the Fourier components of $f(u)$, and where $\varphi_{t_{0}}(s)$ is some smooth function which can be chosen to satisfy for large $|s|$ a bound of the form $\left|\varphi_{t_{0}}(s)\right| \leqslant O\left[\left(1+t_{0}|s|\right)^{-N}\right]$ for as large an $N$ as we wish. The Fourier coefficients are trivially bounded by the $L^{1}$-norm of $f$. The bound (113) then gives, altogether

$$
\begin{aligned}
& \left\|\left(1-P_{2}\right) I^{-1}\left(1+e^{s} \Delta_{1}\right)^{-1} \phi(f) \Omega\right\| \\
& \quad \lesssim\|f\|_{L^{1}}\left(\sum_{n \in \mathbb{Z}} O\left(|\delta n|^{-N}\right) O\left(|n|^{2 h-1}\right)\right)^{\frac{1}{2}} \\
& \quad \lesssim\|f\|_{L^{1}}\left(\frac{1}{\delta}\right)^{h},
\end{aligned}
$$

with implicit constants depending on $s$. We can likewise find a sequence $\left\{Y_{n}\right\}$ in $\mathfrak{M}_{2}$ converging strongly to $\phi(g)$, and thereby obtain a similar result as (114) replacing $\phi(f)$ by $\phi(g)$. Combining these two results now with (45) and using the Cauchy-Schwarz inequality on the right side of that equation gives

$$
\begin{aligned}
& \left|\left\langle\Omega\left|\phi(g)^{*}\left(1+e^{s} \Delta_{1}\right)^{-1} \phi(f)\right| \Omega\right\rangle-\left\langle\Omega\left|\phi(g)^{*}\left(1+e^{s} \Delta_{2}\right)^{-1} \phi(f)\right| \Omega\right\rangle\right| \\
& \quad \lesssim\|f\|_{L^{1}}\|g\|_{L^{1}}\left(\delta_{f} \delta_{g}\right)^{-h} .
\end{aligned}
$$


Letting $f, g$ tend to delta-distributions centered at $u, v$ then by defintion, $\delta_{f} \rightarrow$ dist $(u, \partial A) \lesssim \sum\left|u-a_{j}\right|\left|u-b_{j}\right|$ and likewise for $g$, and the $L^{1}$ norms remain bounded. This gives the claim of the lemma.

From here, we can get:

Lemma 8. Let $u, v \in A=\cup_{i=1}^{p}\left(a_{i}, b_{i}\right)$. Then

$$
|F(\xi, u \mp i 0, v)| \lesssim \sum_{q_{j}}\left|u-q_{j}\right|^{-h}\left|v-q_{j}\right|^{-h}+|u-v|^{-2 h},
$$

with implicit constant depending on $\beta, s$ and the endpoints $\left\{q_{j}\right\}$ of the intervals.

Proof. For definiteness, consider $F(\xi, u-i 0, v)$. From (106), we get for $\Delta>0$ the identity

$$
\frac{1}{\left(\Delta^{-1}+1\right)^{-1}+\xi-\frac{1}{2}}=\frac{1}{\xi+\frac{1}{2}}-\frac{i}{2} \frac{1}{\xi^{2}-\frac{1}{4}} \int_{-\infty}^{\infty} \mathrm{d} t\left(\frac{\xi-\frac{1}{2}}{\xi+\frac{1}{2}}\right)^{i t} \frac{\Delta^{-i t}}{\sinh [\pi(t-i 0)]}
$$

We use this for $\Delta=\Delta_{1}$, the modular operator for the full algebra $\mathfrak{A}$. Then $\Delta_{1}^{i t}$ generates translations by $-\beta t / 2 \pi$ of the coordinate $u$. Now we sandwich the above identity between $\langle\Omega| \phi(u-i 0)$ and $\phi(v+i 0)|\Omega\rangle$. Then if $F_{1}$ is defined as $F$ but with $\Delta_{1}$, we get

$$
\begin{aligned}
F_{1}(\xi, u-i 0, v)= & \frac{1}{\xi+\frac{1}{2}}\langle\Omega \mid \phi(u) \phi(v) \Omega\rangle \\
& -\frac{i}{2} \frac{1}{\xi^{2}-\frac{1}{4}} \int_{-\infty}^{\infty} \mathrm{d} t\left(\frac{\xi-\frac{1}{2}}{\xi+\frac{1}{2}}\right)^{i t} \frac{\langle\Omega \mid \phi(u+\beta(-t-i 0) / 2 \pi) \phi(v) \Omega\rangle}{\sinh [\pi(t-i 0)]} .
\end{aligned}
$$

The second term is uniformly bounded in $u, v$ since the integrand is the boundary value of an analytic function that his holomorphic for $-\varepsilon<\Im(t)<0$ with algebraic singularities. The first term behaves as $\sim(u-v)^{-2 h}$, since this is the UV-behavior of a thermal 2point function of a field of conformal dimension $h$, as one may also prove rigorously by decomposing the field into modes and using (iv) of Assumption 1. The proof now follows by writing $|F| \equiv\left|F_{2}\right| \leqslant\left|F_{2}-F_{1}\right|+\left|F_{1}\right|$ and using the preceeding Lemma 7 on $\left|F_{2}-F_{1}\right|$. We remark that the proof actually shows $F(\xi, u-i 0, v) \sim \frac{e^{-i \pi h}}{2 \pi} \frac{1}{\xi+\frac{1}{2}}(u-v-i 0)^{-2 h}$ for $u$ near $v$ if the field $\phi$ is in standard normalization (24).

Our Assumptions 1 and the fermionic nature of $\phi$ (i.e. $h \in \frac{1}{2} \mathbb{N}$ ) imply the operator expansion in anti-commutator form,

$$
\{\phi(u), \phi(v)\}=\sum_{n=0}^{2 h-1} \delta^{(n)}(u-v) O_{n}\left(\frac{1}{2}(u+v)\right),
$$

as one can see for instance using the relation between our operator algebraic formalism and Vertex Operator Algebras, see [23]. Here the $O_{n}$ are hermitian bosonic primary fields of conformal dimension $2 h-1-n$, and $\delta^{(n)}(u)=\frac{\mathrm{d}^{n}}{\mathrm{~d} u^{n}} \delta(u)$. We let $\left\langle O_{n}\right\rangle=\left\langle\Omega \mid O_{n}(u) \Omega\right\rangle$, 
which is independent of $u$ for our rotation invariant thermal state $|\Omega\rangle$, and use this relation in Lemma 6 , to obtain a new version of the jump condition involving the thermal expectation values $\left\langle O_{n}\right\rangle$.

We summarize the properties of $F(93)$ :

Theorem 3. Let $\phi$ be a fermionic field of conformal dimension $h$ in the normalization (24).

- For fixed $v \in A$ and $\xi \in\left(\frac{1}{2}, \infty\right) \cup\left(-\frac{1}{2},-\infty\right), F(\xi, u, v)$ is a holomorphic function of $u$ on the periodically cut complex plane $\mathbb{C} \backslash(A+\mathbb{Z}+\tau \mathbb{Z})$ with the periodicity $F(\xi, u+1, v)=F(\xi, u+\tau, v)=-F(\xi, u, v)$.

- Across the cuts, $u \in A, F$ satisfies the jump condition

$$
\begin{aligned}
& -\left(\xi+\frac{1}{2}\right) F(\xi, u+i 0, v)+\left(\xi-\frac{1}{2}\right) F(\xi, u-i 0, v) \\
& =\sum_{n=0}^{2 h-1} \delta^{(n)}(u-v)\left\langle O_{n}\right\rangle .
\end{aligned}
$$

- Near the end-points $q_{i}$ of the intervals, $|F| \lesssim\left|q_{i}-u\right|^{-h}$, as a function of $u$.

- Near $v$,

$$
F(\xi, u-i 0, v) \sim \frac{e^{-i \pi h}}{2 \pi} \frac{1}{\xi+\frac{1}{2}}(u-v-i 0)^{-2 h}
$$

as a function of $u$.

- Analogous properties hold true for $u \leftrightarrow v$.

Now we let

$$
G(u-v)=\frac{\vartheta_{1}^{\prime}(\tau)}{2 \pi i \vartheta_{3}(\tau)} \frac{\vartheta_{3}(u-v ; \tau)}{\vartheta_{1}(u-v ; \tau)}
$$

also equal to the thermal 2-point function of the free Fermi field (see below). Our conventions for the Jacobi $\vartheta$ functions are summarized in the appendix. Then the combination $G\left(u-u^{\prime}\right) F\left(\xi, u^{\prime}, v\right)$ is doubly periodic in $u^{\prime}$ for any fixed $v \in A$. Thus, integrating around a contour $\gamma_{\square}$ surrounding the fundamental parallelogram as in fig. 1 , we get zero,

$$
0=\oint_{\gamma_{\square}} \mathrm{d} u^{\prime} G\left(u-u^{\prime}\right) F\left(\xi, u^{\prime}, v\right) .
$$

Since $G\left(u-u^{\prime}\right) \sim \frac{1}{2 \pi i}\left(u-u^{\prime}\right)^{-1}$ for $u \sim u^{\prime}$, and $u=u^{\prime}$ is the only singularity of $G$ inside the fundamental parallelogram, we can deform the contour $\gamma_{\square}$ to a contour tightly surrounding the intervals as shown in fig. 1 , for $\Im(u) \rightarrow 0^{-}$and $\Re(u) \in A$. Then we collect the residue, use the jump condition in the theorem, as well as $\sum_{n=0}^{2 h-1}\left\langle O_{n}\right\rangle G^{(n)}(u-v-i 0)=\langle\Omega \mid \phi(u) \phi(v) \Omega\rangle$. This gives:

Corollary 1. Let $\phi$ be a fermionic primary field of dimension $h . F$ defined as in (93) satisfies (in the distributional sense for $u, v \in A$ ):

$$
\begin{aligned}
\left\langle\Omega_{\beta} \mid \phi(u) \phi(v) \Omega_{\beta}\right\rangle= & -\left(\xi+\frac{1}{2}\right) F(\xi, u-i 0, v) \\
& +\frac{1}{2 \pi i} \int_{A} \mathrm{~d} u^{\prime} \frac{\vartheta_{1}^{\prime}(\tau)}{\vartheta_{3}(\tau)} \frac{\vartheta_{3}\left(u-u^{\prime}-i 0 ; \tau\right)}{\vartheta_{1}\left(u-u^{\prime}-i 0 ; \tau\right)} F\left(\xi, u^{\prime}-i 0, v\right) .
\end{aligned}
$$

Here, $f$ denotes a regulated version of the integral described in the following remark. 


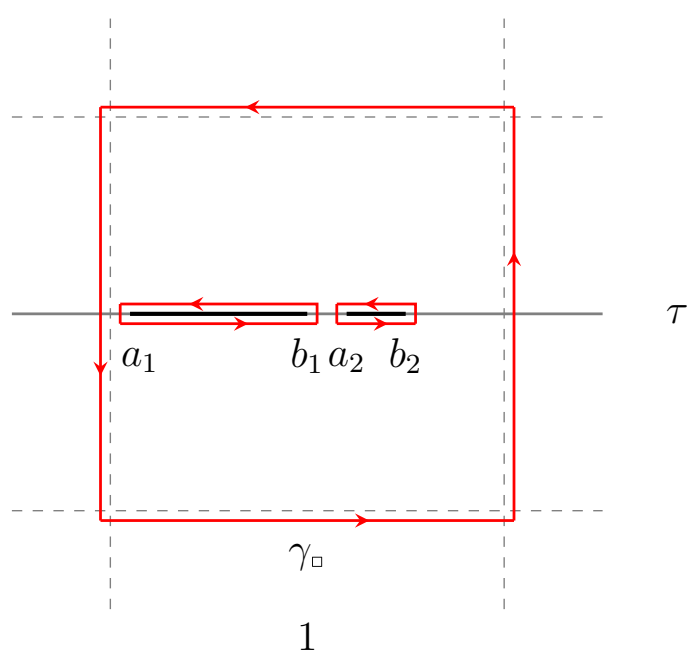

Fig. 1. Illustration of the contour deformation in complex $u$-plane

Remark/Definition: The meaning of the regularized integral operation $f_{A}$ is somewhat subtle because because $F^{ \pm}(\xi, u, v)=F(\xi, u \pm i 0, v)$ are distributions of $u \in \mathbb{R}$ for fixed $v \in A$. As such, there is no obvious meaning to the integral in Corollary 1 . To define $f_{A}$, we must remember the origin of the formula from an integration along a contour fitting tightly around $A$, see fig. 1 . We consider tempered distributions $\widetilde{F}^{ \pm}(\xi, u, v)$ of $u \in \mathbb{R}$ with the property that

(a) $\widetilde{F}^{+}(\xi, u, v)-\widetilde{F}^{-}(\xi, u, v)=F^{+}(\xi, u, v)-F^{-}(\xi, u, v)$,

(b) $\left.\widetilde{F}^{ \pm}(\xi, u, v)\right|_{A^{\prime}}=0$, as a distribution tested with functions of $u$ having compact support in $A^{\prime}$ (thus away from any boundary point), and

(c) $\left.\widetilde{F}^{ \pm}(\xi, u, v)\right|_{A}$ satisfies the jump condition (120) of Theorem 3 as a distribution tested with functions of $u$ having compact support in $A$ (thus away from any boundary point).

We then define the regulated integral by replacing $F^{-}$with $\widetilde{F}^{-}$, now integrated over all $u$, which is now well-defined. Corollary 1 holds with this prescription, since (a), (b), (c) are all that is used about $F$ in the vicinity of the real axis in the above proof.

The existence of $\widetilde{F}^{ \pm}$can be seen as follows. In $A$, it is defined to be equal to $F^{ \pm}$. In $A^{\prime}$, it is defined to zero. This defines distributions for all $u \in \mathbb{R}$, except for the boundary points $q_{i}$. We wish to define $\widetilde{F}^{ \pm}$by a suitable distributional extension. At the boundary points the unextended distributions have "scaling degree" at most $-h$ (by Theorem 3 ), i.e. finite. Therefore, by standard results (see e.g. [38]), an extension exists and is unique up to addition of $\sum_{q_{j}} \sum_{k \leqslant h-1}{ }^{ \pm} A_{j}^{k} \delta^{(k)}\left(u-q_{j}\right)$. The free parameters must be adjusted in such a way that (c) holds for all $u \in \mathbb{R}$, not just inside $A$ away from the boundary points, and such that $(a)$ still holds. This uniquely determines $\widetilde{F}^{ \pm}$, and hence our prescription $f_{A} \cdot{ }^{7}$

The corollary imposes on $F$ an integral equation at which we will look at in more detail in the following example. The example also suggests that corollary 1 can be used to find the modular operator in the case of a general fermionic primary field of dimension $h$.

\footnotetext{
7 We remark that in the expressions considered below in Sect. 5.4, our regulated integral has the same meaning as that explained in sec. 4 of [11].
} 
5.2. Example: Modular Hamiltonian for thermal state of free Fermi field. Now we analyze what we can learn from Theorem 3 and Corollary 1 for the free Fermi field, where $h=\frac{1}{2}$ and $\{\psi(u), \psi(v)\}=\delta(u-v) 1$. For this theory, we can actually prescribe thermal states (92) using either the Neveu-Schwarz or the Ramond representations. For the Neveu-Schwarz (vacuum) sector, the representation is the half-integer moded expansion (29), now denoted as $\psi_{\mathrm{NS}}(z)$. For the Ramond-sector, the representation corresponds to an integer moded expansion given in (32) now denoted as $\psi_{\mathrm{R}}(z)$. The Gibbs states (92) in the Neveu-Schwarz/Ramond representation correspond, accordingly, to the thermal state vectors $\left|\Omega_{\mathrm{NS}, \beta}\right\rangle$ and $\left|\Omega_{\mathrm{R}, \beta}\right\rangle$, respectively. The thermal 2-point functions are [39]:

$$
\left\langle\Omega_{\mathrm{X}, \beta} \mid \psi_{\mathrm{X}}(u) \psi_{\mathrm{X}}(v) \Omega_{\mathrm{X}, \beta}\right\rangle=G_{\mathrm{X}}(u-v-i 0)
$$

where the subscript $X \in\{R, N S\}$ indicates the choice of boundary conditions (sector). For NS, the definition was already given above in (126), whereas for R, we set

$$
G_{\mathrm{R}}(u-v)=\frac{\vartheta_{1}^{\prime}(\tau)}{2 \pi i \vartheta_{2}(\tau)} \frac{\vartheta_{2}(u-v ; \tau)}{\vartheta_{1}(u-v ; \tau)}
$$

Next we define $F_{X}$ as in (93). By Theorem 3 we know its properties, with the only trivial difference that $F_{\mathrm{R}}(s, u+1, v)=F_{\mathrm{R}}(\xi, u, v)$ in the Ramond sector, where the subscript $\mathrm{X} \in\{\mathrm{R}, \mathrm{NS}\}$ indicates the choice of boundary conditions. These properties uniquely determine $F_{\mathrm{X}}$ in our case (cf. table 1):

$$
\begin{aligned}
F_{\mathrm{R}, \mathrm{NS}}(\xi, u, v)= & \frac{1}{\xi+\frac{1}{2}} \frac{\vartheta_{1}^{\prime}(\tau)}{2 \pi i \vartheta_{1}(u-v ; \tau)} \frac{\vartheta_{2,3}(u-v-i s|A| / 2 \pi ; \tau)}{\vartheta_{2,3}(-i s|A| / 2 \pi ; \tau)} \\
& \times\left(\frac{\Pi_{a}(u ; \tau) \Pi_{b}(v ; \tau)}{\Pi_{b}(u ; \tau) \Pi_{a}(v ; \tau)}\right)^{i s / 2 \pi},
\end{aligned}
$$

assuming for instance $-\varepsilon<\Im(u)<0$. Here $|A|=\sum_{j}\left(b_{j}-a_{j}\right)$ is the total length of the intervals,

$$
e^{s}=\frac{\xi+\frac{1}{2}}{\xi-\frac{1}{2}}
$$

and

$$
\Pi_{a}(u ; \tau)=\prod_{i=1}^{p} \vartheta_{1}\left(u-a_{i} ; \tau\right)
$$

etc.

By the well-known periodicity properties and pole/zero structure of the $\vartheta$-functions, it is easy to check that the above formula indeed satisfies the the properties known to hold by Theorem 3. For instance, the role of the second line is to give the required jump across $A$, and the role of the pole at $u=v$ in the first line gives the delta-function in (117), also consistent with (142). To show that $F$ as given is the only solution to the properties in Theorem 3, we multiply any solution $F$ with the inverse of the second line. This will cancel the jump of $F$ except for the delta function in (117). Thus, the multiplied $F$ can have a first order pole at $u=v \bmod \mathbb{Z}$ with residue $1 /\left(\xi-\frac{1}{2}\right)$, by (117). It cannot have poles, however, at the end-points $q_{i}$ of the intervals, since Theorem 3 tells us that $|F| \lesssim\left|q_{i}-u\right|^{-1 / 2}$. Thus, the multiplied function $F$ is meromorphic with a single pole 
in the fundamental parallelogram, with the same residue and periodicity properties as the first line. There can only be one such function, by well-known results on elliptic functions.

Knowing $F$, we get the kernel of the modular hamiltonian $\left\langle\Omega_{\mathrm{X}, \beta}\right| \psi_{\mathrm{X}}(u)(\ln \Delta) \psi_{\mathrm{X}}(v)$ $\left.\Omega_{X, \beta}\right\rangle$ by integrating up (96). Thus, we have, in principle, found this kernel. The (nontrivial) integration over $\xi$ in (96) has recently performed by $[15,16]$, who have independently arrived at an analogous formula as for $F$ for a closely related quantity, by a different method based on resolvents. It is instructive to see more precisely how this method is related to ours, as it may also shed light on how to proceed in the case of fields with general conformal dimension $h$.

For this purpose, let us set $G_{X}(u-v)$ be the thermal 2-point function given by (125), with $\mathrm{X} \in\{\mathrm{NS}, \mathrm{R}\}$. By Theorem $3, F_{\mathrm{X}}$ has the same periodicity as $G_{\mathrm{X}}$, so the combination $G_{\mathrm{X}}\left(u-v^{\prime}\right) F_{\mathrm{X}}(\xi, u, v)$ is doubly periodic in $u$ for any fixed $v \in A$. Thus, integrating again around a contour $\gamma_{\square}$ surrounding the fundamental parallelogram as in fig. 1, we get zero as in (123), with $G=G_{X}$ now in that equation. Such an identity also derived by [16] via a different argument using the special properties of the free Fermi field. By contrast, we have so far only used general properties and Theorem 3, so our argument works for any CFT.

Now, we deform again the contour $\gamma \square$ to a contour tightly surrounding the cuts $A$ inside the fundamental parallelogram, see fig. 1, use the jump properties and bounds on $F$ as given in Theorem 3 and collect the residue. While these steps can still be performed in any CFT according to Theorem 3, the free Fermi case is especially simple because the poles of $F$ near the end-points $q_{i}$ of the intervals are of the order $\left|u-q_{i}\right|^{\frac{1}{2}}$, and hence integrable. Thereby, we immediately get the integral equation

$$
\begin{aligned}
& G_{\mathrm{X}}(u-v-i 0) \\
& \quad=-\left(\xi+\frac{1}{2}\right) F_{\mathrm{X}}(\xi, u-i 0, v)+\int_{A} \mathrm{~d} u^{\prime} G_{X}\left(u-u^{\prime}-i 0\right) F_{X}\left(\xi, u^{\prime}-i 0, v\right)
\end{aligned}
$$

without any need for a regulator in the integral as in Corollary 1. Dropping the subscript "X" for ease of notation and indicating by $\left.G_{A} \equiv G\right|_{A}$ the restriction of the kernels (125) to $A \times A$, we may view $G_{A}$ as an operator $L^{2}(A) \rightarrow L^{2}(A)$. In fact, the anti-commutation relations and the positive nature of the Hilbert space inner product in (125) imply the operator inequalities $0 \leqslant G_{A} \leqslant 1$. Now we define $F_{A}^{ \pm}(\xi)$ the operator defined by the restriction $F(\xi, u \pm i 0, v)$ to $A$ in kernel notation, i.e. when $u, v \in A$. We can then also write the above integral equation in operator notation as $G_{A}=\left(G_{A}-\xi-\frac{1}{2}\right) F_{A}^{-}(\xi)$ and solve it as

$$
F_{A}^{-}(\xi)=\left(G_{A}-\xi-\frac{1}{2}\right)^{-1} G_{A}
$$

when $\xi \in\left(\frac{1}{2}, \infty\right) \cup\left(-\frac{1}{2},-\infty\right)$. In view of (96), we should then further calculate, in operator notation

$$
\int_{\frac{1}{2}}^{\infty} \mathrm{d} \xi\left(F_{A}^{-}(\xi)+F_{A}^{-}(-\xi)\right)=\ln \left(G_{A}^{-1}-1\right) G_{A} .
$$

Thus, as a kernel, the modular hamiltonian is given by

$$
\langle\Omega \mid \psi(u)(\ln \Delta) \psi(v) \Omega\rangle=-\left(\ln \left(G_{A}^{-1}-1\right) G_{A}\right)(u, v) .
$$


Formally, this may also be expressed as saying $H_{A}=\frac{1}{2} \int_{A \times A} k_{A}(x, y) \psi(x) \psi(y) \mathrm{d} x \mathrm{~d} y$ and $k_{A}=\ln \left(G_{A}^{-1}-1\right)$ is the hamiltonian appearing in eq. (80). This is in accord with a well known formula [14] for the modular Hamiltonian of the free Fermi field in terms the restricted 2-point function $G_{A}$, which can be derived using the Fock-space structure of theory. In the present case, however, our derivation was rather different and can be paralleled for fermionic fields in general CFTs which are not necessarily equivalent to free field theories. As derived here, our formula holds for thermal states in either the NS or the R sector, choosing for $G$ either one of the 2-point functions (125). Thus, apart from confirming our method, this relation to integral equations might also be useful in the case of more general CFTs where one does not have a priori relations like (133).

5.3. Bosonic fields. We now repeat a similar analysis for a bosonic field $\phi$ of dimension $h \in \mathbb{N}$, satisfying our standing Assumptions 1. In fact, most results in Sect. 5.1 for fermions hold just as well for bosons, with nearly identical proof. A difference, however, arises in the jump condition (98), which involves the anti-commutator of $\phi$, whereas for bosons, we would like to have the commutator. This means that we should work with a different definition of $F,(93)$. In the bosonic case, we instead define using the shorthands $|\Omega\rangle \equiv\left|\Omega_{\beta}\right\rangle$ and $\Delta \equiv \Delta_{\beta, A}$

$$
F(s, u, v)= \begin{cases}\left\langle\Omega \mid \phi(u)\left[1-e^{s}(1-\Delta)^{-1}\right]^{-1} \phi(v) \Omega\right\rangle & \text { if }-\beta<\Im(u)<0, \\ \left\langle\Omega \mid \phi(v)\left[1-e^{s}\left(1-\Delta^{-1}\right)^{-1}\right]^{-1} \phi(u) \Omega\right\rangle & \text { if } \beta>\Im(u)>0 .\end{cases}
$$

Since $\Delta>0, F$ is well defined, in the distributional sense if $s>0$, and we refrain from using a different symbol for $F$ even though it is a different quantity compared to Sect. 5.1. The connection to the modular Hamiltonian now follows from the elementary formula

$$
\ln \Delta=\int_{0}^{\infty} \mathrm{d} s \frac{1}{1-e^{s}\left(1-\Delta^{-1}\right)^{-1}},
$$

giving for $\Im(u)>0$, say,

$$
\langle\Omega \mid \phi(v)(\ln \Delta) \phi(u) \Omega\rangle=\int_{0}^{\infty} \mathrm{d} s F(s, u, v) .
$$

Thus, we should determine $F$. Again, the key result is a lemma expressing a jump condition.

Lemma 9. Let $v \in A$ be fixed. If $u \in A^{\prime}$, then

$$
F(s, u-i 0, v)=F(s, u+i 0, v) .
$$

If $u \in A$, then

$$
\left(1-e^{s}\right) F(s, u-i 0, v)-F(s, u+i 0, v)=\langle\Omega \mid[\phi(u), \phi(v)] \Omega\rangle,
$$

in the sense of distributions. Here $[\phi(u), \phi(v)]=\phi(u) \phi(v)-\phi(v) \phi(u)$ is the commutator.

Proof. Analogous to the proof of Lemma 6. 
Our Assumption 1 and the bosonic nature of $\phi$ imply the operator expansion

$$
[\phi(u), \phi(v)]=\sum_{n=0}^{2 h-1} \delta^{(n)}(u-v) O_{n}\left(\frac{1}{2}(u+v)\right),
$$

which may be used to eliminate the commutator in the jump condition (138). Certain obvious changes also apply to the periodicity of $F$, which is now (103) without the "-" sign. In the proof of Lemma 8 we now use the formula

$$
\frac{1}{1-e^{s}\left(1-\Delta^{-1}\right)^{-1}}=1-\frac{i}{2} \frac{1}{e^{s}-1} \int_{-\infty}^{\infty} \mathrm{d} t \frac{\left[\left(e^{s}-1\right) \Delta\right]^{i t}}{\sinh [\pi(t-i 0)]} .
$$

instead of (117), leading now to $F(s, u-i 0, v) \sim \frac{e^{-i \pi h}}{2 \pi}(u-v-i 0)^{-2 h}$ for $u$ near $v$. Altogether, this gives the following variant of Theorem 3 for bosonic fields:

Theorem 4. - For fixed $v \in A$ and $s>0, F(s, u, v)$ is a holomorphic function of $u$ on the periodically cut complex plane $\mathbb{C} \backslash(A+\mathbb{Z}+\tau \mathbb{Z})$ with the periodicity $F(s, u+1, v)=F(s, u+\tau, v)=F(s, u, v)$.

- Across the cuts, $u \in A, F$ satisfies the jump condition

$$
\left(1-e^{s}\right) F(s, u-i 0, v)-F(s, u+i 0, v)=\sum_{n=0}^{2 h-1} \delta^{(n)}(u-v)\left\langle O_{n}\right\rangle .
$$

- Near the end-points $q_{i}$ of the intervals, $|F| \lesssim\left|q_{i}-u\right|^{-h}$, as a function of $u$.

- Near $v$,

$$
F(s, u-i 0, v) \sim \frac{e^{-i \pi h}}{2 \pi}(u-v-i 0)^{-2 h}
$$

as a function of $u$.

- Analogous properties hold true for $u \leftrightarrow v$.

Remark 3. We will see below that the theorem, or alternatively the following Corollary 2, may be used to find $F$, and thereby the modular Hamiltonian in view of (136). See Sect. 5.4 for examples.

In the case of bosonic fields, it is apparently not straightforward to get an analog of the integral equation in Corollary 1 . The problem is that $G$, the analogue of (126) should have a simple pole at $u=v$ and be such that $G\left(u-u^{\prime}\right) F\left(s, u^{\prime}, v\right)$ is doubly periodic in $u^{\prime}$ for fixed $v \in A$. Since, by contrast to fermionic fields, $F(s, u, v)$ is itself doubly periodic in $u$, the simplest choice-in the absence of any other structural properties of $F$, would be to choose $G$ as doubly periodic, too. However, as is well known there is no such meromorphic function with only one simple pole in the fundamental parallelogram. We will, however, be able to make a similar construction below in the case of the $U(1)$ current, where $F$ has an additional property.

In the general case, we can nevertheless still find an integral equation in the vacuum case, where we can simply set $G(x-y)=(2 \pi i)^{-1}(x-y)^{-1}$. Here, we go back to the real line picture in which the theory is living on $\mathbb{R}$ parameterized by $x, y$, and we correspondingly write $F(s, x, y)$, etc. Theorem 4 still applies to the vacuum case: By inspection of the proof, we can take in the end the limit $\beta \rightarrow \infty$. A similar type of argument as for Corollary 1 now leads to (using $\left\langle O_{n}\right\rangle=0$ except when $O_{n}=1$ in the vacuum state): 


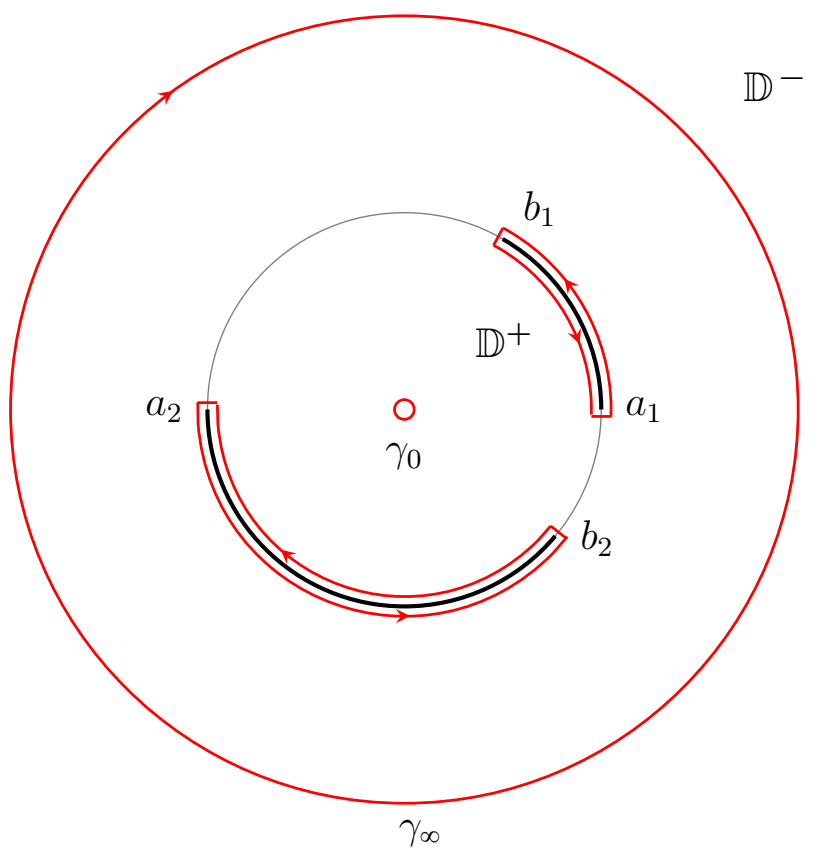

Fig. 2. Illustration of the contour deformation in the complex $y^{\prime}$-plane

Corollary 2. $F$ defined as in (134) (for a bosonic primary field $\phi$ of dimension $h$ in standard nomalization (24)) satisfies for the vacuum state $|\Omega\rangle \equiv\left|\Omega_{0}\right\rangle$ :

$$
\begin{aligned}
\left\langle\Omega_{0} \mid \phi(x) \phi(y) \Omega_{0}\right\rangle= & F(s, x, y+i 0) \\
& +e^{s} \frac{1}{2 \pi i} \int_{A} \mathrm{~d} y^{\prime} F\left(s, x, y^{\prime}+i 0\right)\left(y^{\prime}-y-i 0\right)^{-1} .
\end{aligned}
$$

for $x, y \in A \subset \mathbb{R}$ (lightray picture).

Proof. For the proof, we first work in the circle picture where $x, y \in \mathbb{S}$. For our assumptions about the fields, we have for $z \in \mathbb{D}^{+}=\{z:|z|<1\}$ that $\phi(z)\left|\Omega_{0}\right\rangle=$ $\sum_{n \geqslant h} z^{n-h} \phi_{n}\left|\Omega_{0}\right\rangle$ which is holomorphic in $\mathbb{D}^{+}$. Likewise, for $z \in \mathbb{D}^{-}=\{z:|z|>1\}$ that $\left\langle\Omega_{0}\right| \phi(z)=\sum_{n \geqslant h} z^{-n-h}\left\langle\phi_{n} \Omega_{0}\right|$ which is holomorphic in $\mathbb{D}^{-}$and goes as $|z|^{-2 h}$ for $|z| \rightarrow \infty$. This implies that, for fixed $x \in A, F(s, x, y)$ is a holomorphic function in $\mathbb{C} \backslash A$ decaying like $|y|^{-2 h}$ for $|y| \rightarrow \infty$. Now we consider $y \in \mathbb{D}^{+}$, a small contour $\gamma_{0}$ around $y^{\prime}=0$, and the identity

$$
\frac{1}{2 \pi i} \oint_{\gamma_{0}} \mathrm{~d} y^{\prime} F\left(s, x, y^{\prime}\right)\left(y^{\prime}-y\right)^{-1}=0 .
$$

We then move the contour $\gamma_{0}$ across the cuts $A$ as in fig. 2, deform it to a very large circle $\gamma_{\infty}$, use the decay of $F\left(s, x, y^{\prime}\right)$ as well as the jump conditions (141) across the cuts $A$ and collect the residue. The statement then follows after transforming back to the lightray picture. 
5.4. Example: Modular Hamiltonian of free $U(1)$-current. The $U(1)$ current, $j$, was introduced in example 6 above. It has dimension $h=1$ and commutator $[j(u), j(v)]=$ $i \delta^{\prime}(u-v)$. The thermal 2-point function is

$$
\left\langle\Omega_{\beta} \mid j(u) j(v) \Omega_{\beta}\right\rangle=-\frac{1}{2 \pi}\left(\wp(u-v-i 0 ; \tau)-\eta_{1}(\tau)\right) \equiv G(u-v-i 0) .
$$

Here, $\eta_{1}, \eta_{2}$ are the constants appearing in connection with the Weierstrass $\wp$-function, see appendix.

Guessing a-hopefully unique — answer for $F$ just from the properties given in Theorem 4 as in the case of the free Fermi field does not seem as straightforward for the current, so we proceed by the more deductive method of integral equations. We have already mentioned that for thermal states, we have not found a general way to obtain an analogue of Corollary 1 for bosons. However, for the case of the U(1)-current, there is an additional structural property which helps.

Consider the Weierstrass $\zeta$-function satisfying $\zeta^{\prime}=-\wp$, see appendix. It has a single simple pole at $u=0$ in the fundamental parallelogram but is only quasi-periodic, $\zeta(u+1)=\zeta(u)+\eta_{1}, \zeta(u+\tau)=\zeta(u ; \tau)+\eta_{2}$. As a consequence, the function $\zeta(u)-\eta_{1} u$ is periodic under $u \rightarrow u+1$, and changes by $\eta_{2}-\eta_{1} \tau=-2 \pi i$ under $u \rightarrow u+\tau$. The combination $\left\{\zeta\left(v^{\prime}-v\right)-\left(v^{\prime}-v\right) \eta_{1}\right\} F\left(\xi, u, v^{\prime}\right)$ is also not doubly periodic in $v^{\prime}$ for any fixed $u \in A$. However, integrating around a contour $\gamma \square$ surrounding the fundamental parallelogram as in fig. 1 ,

$$
0=\oint_{\gamma_{\square}} \mathrm{d} u F\left(s, u, v^{\prime}\right)\left\{\zeta\left(v^{\prime}-v\right)-\left(v^{\prime}-v\right) \eta_{1}\right\},
$$

still gives zero because of the special property of $F$ that, for $u \in A$,

$$
\begin{aligned}
\int_{0}^{1} F\left(s, u, v^{\prime}+\varepsilon\right) \mathrm{d} v^{\prime} & = \begin{cases}\left\langle\left[1-e^{s}(1-\Delta)^{-1}\right]^{-1} j(u) \Omega \mid j_{0} \Omega\right\rangle & \text { if }-\beta<\Im(\varepsilon)<0, \\
\left\langle j_{0} \Omega \mid\left[1-e^{s}\left(1-\Delta^{-1}\right)^{-1}\right]^{-1} j(u) \Omega\right\rangle & \text { if } \beta>\Im(\varepsilon)>0,\end{cases} \\
& =0,
\end{aligned}
$$

which follows from the mode expansion of $j(v)=\sum_{n=-\infty}^{\infty} j_{n} e^{-2 \pi i n v}$ and the fact that $j_{0}$ is the charge operator, which annihilates any state in the vacuum sector. Hence also $j_{0}|\Omega\rangle=0$ for our thermal state, since it can be viewed as a statistical operator on the vacuum Hilbert space.

If we now evaluate this contour integral as before taking the properties of $F$ in Theorem 4 into account, then we get

$$
\begin{aligned}
& \frac{1}{2 \pi}\left\{\wp(u-v-i 0)-\eta_{1}\right\}=F(s, u, v+i 0) \\
& \quad+\frac{1}{2 \pi i} e^{s} f_{A} \mathrm{~d} v^{\prime} F\left(s, u, v^{\prime}+i 0\right)\left\{\zeta\left(v^{\prime}-v-i 0\right)-\left(v^{\prime}-v\right) \eta_{1}\right\} .
\end{aligned}
$$

Here, $f$ denotes the regulated integral described in and below Corollary 1 .

We now consider the connection with formulae in the literature for the kernel of the modular Hamiltonian. First, we decompose

$$
G(u-v-i 0)=S(u-v)+\frac{i}{2} C(u-v),
$$


with $\frac{i}{2} C(u-v)=\delta^{\prime}(u-v)$ symmetric (imaginary) and $S(u-v)=-\frac{1}{2 \pi}$ P.V. $(\wp(u-$ $v)-\eta_{1}$ ) the symmetric (real) part of $G$ as in (145) (P.V. means Cauchy principal value). Since the correlation function satisfies $\left\langle\Omega \mid j(f)^{*} j(f) \Omega\right\rangle \geqslant 0$, we get $G(\bar{f}, f) \geqslant 0$ for any test-function $f \in C_{0}^{\infty}(A, \mathbb{C})$. Decomposing $f=g+i h$ into a real and imaginary part, we get

$$
\frac{1}{2}|C(h, g)| \leqslant \sqrt{S(h, h)} \sqrt{S(g, g)}
$$

for all $g, h \in C_{0}^{\infty}(A, \mathbb{R})$. We then use the positive definite $\mathbb{R}$-bi-linear form $S$ to define on $C_{0}^{\infty}(A, \mathbb{R})$ an inner product, which we then extend to a hermitian inner product by complex anti-linearity in the first entry. Let this hermitian positive definite sesqui-linear form be called $(,)_{S}$. Its completion defines a Hilbert space, $\mathcal{K}_{A}$, which is contained in the Sobolev space $W_{0}^{1 / 2,2}(A)$. It is the 1-particle space of the GNS-Fock space built on $|\Omega\rangle \equiv\left|\Omega_{\beta}\right\rangle$. It follows from the definitions that $\mathcal{K}_{A} \ni f \mapsto \phi(f)|\Omega\rangle \in \mathcal{H}$ is an isometry. Furthermore, since $\left[1-e^{s}\left(1-\Delta^{ \pm 1}\right)^{-1}\right]^{-1}$ is a bounded, self-adjoint operator on $\mathcal{H}$ for $s>0$, it follows from (134) that the kernel $F^{ \pm}(s, x, y)=F(s, x \pm i 0, y)$ extends to a bounded quadratic form on $\mathcal{K}_{A}$, and hence can be identified with a bounded linear operator on $\mathcal{K}_{A}$.

By Riesz' theorem, there is a self-adjoint operator on this Hilbert space, $\Sigma_{A}$, such that $\frac{i}{2} C(f, g)=\left(f, \Sigma_{A} g\right)_{S}$ for all $f, g \in \mathcal{K}_{A}$. This operator satisfies $\left|\Sigma_{A}\right| \leqslant 1$, so $\left|\Sigma_{A}^{-1}\right| \geqslant 1$ in view of (150). It follows that for $s>0,\left(e^{-s}-\frac{1}{2}+\frac{1}{2} \Sigma_{A}^{-1}\right)^{-1}$ exits and is a bounded, self-adjoint operator on $\mathcal{K}_{A}$. By construction, the operator $\Sigma_{A}$ is expressible as $(i / 2) S_{A}^{-1} C_{A}$, with $S_{A}, C_{A}, G_{A}$ the operators defined by the restriction of the kernels to $A$. Alternatively,

$$
-i C_{A}^{-1} G_{A}=\frac{1}{2}\left(\Sigma_{A}^{-1}-1\right) .
$$

Using $C=\delta^{\prime}$ and following the same argument as in sec. 4 of [11] (where the vacuum state was considered), we find that

$$
C_{A}^{-1} f(u)=\frac{1}{2} \int_{a}^{u} f\left(u^{\prime}\right) \mathrm{d} u^{\prime}-\frac{1}{2} \int_{u}^{b} f\left(u^{\prime}\right) \mathrm{d} u^{\prime}
$$

in the case of one interval $A=(a, b)$, leading to

$$
\begin{aligned}
2 \pi C_{A}^{-1} G_{A}= & \zeta(u-v-i 0)-u \eta_{1}-\frac{1}{2}[\zeta(a-v-i 0) \\
& \left.+\zeta(b-v-i 0)-(a+b) \eta_{1}\right] .
\end{aligned}
$$

Note that the terms in $[\ldots]$ do not depend on $u$, and therefore could be added in the integral (146) in view of (147). Therefore, we can write our integral equation (148) in operator form also as:

$$
F_{A}^{+}(s)\left[1+\frac{1}{2} e^{s}\left(\Sigma_{A}^{-1}-1\right)\right]=-G_{A}
$$

where $F_{A}^{+}(s)$ is the bounded operator on $\mathcal{K}_{A}$ corresponding to the kernel $F(s, u, v+i 0)$. Since we already know that the operator on the right side can be inverted for $s>0$, it follows that

$$
F_{A}^{+}(s)=-G_{A}\left[1+\frac{1}{2} e^{s}\left(\Sigma_{A}^{-1}-1\right)\right]^{-1}
$$


is a solution to our integral equation in operator notation. Integrating this operator identity over $s$ as in eq. (136), we obtain

$$
\begin{aligned}
\int_{0}^{\infty} \mathrm{d} s F_{A}^{+}(s) & =-G_{A} \int_{0}^{\infty} \mathrm{d} s\left[1+\frac{1}{2} e^{s}\left(\Sigma_{A}^{-1}-1\right)\right]^{-1} \\
& =G_{A} \int_{0}^{1} \mathrm{~d} \lambda\left[\lambda+\frac{1}{2}\left(\Sigma_{A}^{-1}-1\right)\right]^{-1} \\
& =G_{A} \ln \left[\frac{\Sigma_{A}^{-1}+1}{\Sigma_{A}^{-1}-1}\right],
\end{aligned}
$$

and thereby in view of eq. (136)

$$
\langle\Omega \mid j(u)(\ln \Delta) j(v) \Omega\rangle=\left(G_{A} \ln \left[\frac{\Sigma_{A}^{-1}+1}{\Sigma_{A}^{-1}-1}\right]\right)(u, v) .
$$

This formula is sometimes quoted in the literature [13] for the matrix elements of the modular Hamiltonian of a free boson field, and should be thought of as analogous to (133) for the fermion field. A similar derivation also goes through if $A=\cup_{i}\left(a_{i}, b_{i}\right)$ consists of an arbitrary number of intervals. By contrast to the results in the literature, our derivation is based on the rigorously derived integral equations, rather than a formal analog with finite-dimensional quantum systems.

We now sketch how to find an explicit expression for the matrix element (157) of the modular Hamiltonian in the case of one interval. To calculate the logarithm of operators, we need the spectral decomposition of the self-adjoint operator $\Sigma_{A}^{-1}$, which we write as

$$
\Sigma_{A}^{-1}=-\int_{-\infty}^{\infty} \mathrm{d} s \operatorname{coth}(s) U_{s}(u) V_{s}(v)
$$

Here, $V_{s}$ is a generalized ${ }^{8}$ left-eigenfunction of $\Sigma_{A}^{-1}$ with eigenvalue $V_{s} \Sigma_{A}^{-1}=-\operatorname{coth}(s)$ $V_{s}$, and $U_{s}$ satisfies $-f_{a}^{b} V_{s}(u) U_{s^{\prime}}(u) \mathrm{d} u=\delta\left(s-s^{\prime}\right)$. Paralleling the steps in sec. 4 of [11], one can see that ( $q_{i}$ ranging over the endpoints $a, b$ of the interval)

$$
V_{s}(u)=\sum_{q_{i}} \frac{c_{q_{i}}(s) \vartheta_{1}\left(u-q_{i}+i s(b-a) / 2 \pi\right)}{\vartheta_{1}\left(u-q_{i}\right)}\left(-\frac{\vartheta_{1}(u-a)}{\vartheta_{1}(u-b)}\right)^{-i s / 2 \pi}
$$

with boundary value prescription $u+i 0$ understood, is such a generalized eigenfunction. This eigenfunction is constructed in such a way that is has a multiplicative jump by $e^{s}$ across the interval $(a, b)$ (from the last term), and such that it is doubly periodic in $u$ (cf. Table 1). Furthermore, near any one $q_{i}$ of the endpoints $a, b$, it is bounded by $\lesssim\left|u-q_{i}\right|^{-1}$ in accordance with Theorem 4 . The constants $c_{q_{i}}(s)$ are at first adjusted so that

$$
\int_{0}^{1} V_{s}(u-i 0) \mathrm{d} u=0 .
$$

Then, we have again the relation (146) with $V_{s}$ in place of $F$, and evaluating this contour integral by deforming the contour around the fundamental parallelogram to a tight

\footnotetext{
8 More precisely, $V_{S}$ is a linear form on a domain $\mathcal{D} \subset \mathcal{K}_{A}$ defined by $g \mapsto f_{A} V_{S}(u) g(u) \mathrm{d} u$.
} 
contour around $(a, b)$ as in fig. 1 then gives the desired eigenvalue equation. That (160) impose no actual loss of generality is demonstrated in the end.

Property (160) is evidently equivalent to

$$
\sum_{q_{i}} c_{q_{i}}(s) I_{q_{i}}(s)=0
$$

where

$$
I_{q}(s)=\int_{a}^{b} \mathrm{~d} u \frac{\vartheta_{1}(u-q+i s(b-a) / 2 \pi)}{\vartheta_{1}(u-q)}\left(-\frac{\vartheta_{1}(u-a)}{\vartheta_{1}(u-b)}\right)^{-i s / 2 \pi} .
$$

The constants $c_{q_{i}}(s)$ can further be adjusted so that $-i \operatorname{sgn}(s) C_{A}^{-1} \bar{V}_{s}=U_{s}$ is a righteigenvector of $\Sigma_{A}^{-1}$, satisfying the desired normalization $\int_{a}^{b} V_{s}(u) U_{s^{\prime}}(u) \mathrm{d} u=\delta\left(s-s^{\prime}\right)$, see sec. 3 of [11] for further discussion of this point. The $\delta$-function in this expression can only come from the the contribution of the integrals near the boundary points $q_{i}$, which gives a practical way of evaluating these constraints.

Using $\ln [(\operatorname{coth}(s)-1) /(\operatorname{coth}(s)+1)]=-2 s$ and $\Sigma_{A}^{-1} U_{s}=-\operatorname{coth}(s) U_{s}$, it immediately follows that

$$
-\frac{i}{2} \ln \left[\frac{\Sigma_{A}^{-1}+1}{\Sigma_{A}^{-1}-1}\right] C_{A}^{-1}=\int_{-\infty}^{\infty} \mathrm{d} s|s| U_{S}(u) \overline{U_{s}(v)}=: k_{A}(u, v) .
$$

Therefore in view of (157) and the commutation relation $[j(u), j(v)]=i \delta^{\prime}(u-v)$, the final answer may also be (formally) rewritten as

$$
\langle\Omega| j(u)(\ln \Delta, j(v) \Omega\rangle=\left\langle\Omega \mid j(u)\left[H_{A}, j(v)\right] \Omega\right\rangle,
$$

where $H_{A}=\int_{A \times A} k_{A}(u, v) j(u) j(v) \mathrm{d} u \mathrm{~d} v$ and $k_{A}(u, v)$ the kernel of the operator on the right side of (163). We refrain here form analyzing in detail the remaining integrals.

This construction can be generalized to the case of $p$ intervals $A=\cup_{j=1}^{p}\left(a_{j}, b_{j}\right)$ in the following way. The ansatz for the generalized eigenfunction is now

$$
V_{s}(u)=\sum_{q_{i}} \frac{c_{q_{i}}(s) \vartheta_{1}\left(u-q_{i}+i s|A| / 2 \pi\right)}{\vartheta_{1}\left(u-q_{i}\right)}\left(-\frac{\prod_{j} \vartheta_{1}\left(u-a_{j}\right)}{\prod_{j} \vartheta_{1}\left(u-b_{j}\right)}\right)^{-i s / 2 \pi},
$$

where $|A|=\sum_{j}\left(b_{j}-a_{j}\right)$. On the $2 p$ coefficients $c_{q_{i}}(s)$ we impose (160) and the $p$ constraints

$$
\int_{a_{j}}^{b_{j}} V_{s}(u) \mathrm{d} u=0, \quad j=1, \ldots, p .
$$

Of these, only $p-1$ are independent because (160) and the periodicity of $V_{s}(u)$ can be used to show that $\sum_{j} f_{a_{j}}^{b_{j}} V_{s}(u) \mathrm{d} u=0$ by integrating $V_{s}(u)$ around a contour as in fig. 1. A priori, (166), (166) are again a restriction on the possible set of eigenfunctions. But in the end one shows that, in fact, all eigenfunctions of $\Sigma_{A}^{-1}$ satisfy this constraint. They are equivalent to

$$
\sum_{q_{i}} c_{q_{i}}(s) I_{q_{i}}^{j}(s)=0, \quad j=1, \ldots, p,
$$


where

$$
I_{q}^{j}(s)=\int_{a_{j}}^{b_{j}} \mathrm{~d} u \frac{\vartheta_{1}(u-q+i s|A| / 2 \pi)}{\vartheta_{1}(u-q)}\left(-\frac{\prod_{j} \vartheta_{1}\left(u-a_{j}\right)}{\prod_{j} \vartheta_{1}\left(u-b_{j}\right)}\right)^{-i s / 2 \pi} .
$$

This leaves us with $p$ linearly independent left-eigenfunctions, $V_{s}^{k}(u), k=1, \ldots, p$. The constants $c_{q_{i}}(s)$ are further be adjusted so that $-i \operatorname{sgn}(s) C_{A}^{-1} \overline{V_{s}^{k}}=U_{s}^{k}$ or equivalently

$$
U_{s}^{k}(u)=-i \operatorname{sgn}(s) \int_{a_{j}}^{u} \mathrm{~d} u^{\prime} \overline{V_{s}^{k}\left(u^{\prime}\right)}, \quad \text { for } u \in\left(a_{j}, b_{j}\right),
$$

is a right-eigenvector of $\Sigma_{A}^{-1}$, satisfying the desired ortho-normalization $\int_{A} V_{s}^{k}(u) U_{s^{\prime}}^{k^{\prime}}(u)$ $\mathrm{d} u=\delta_{k k^{\prime}} \delta\left(s-s^{\prime}\right)$.

Following an argument given in sec. 5 of [11], let us finally explain why conditions (160), (166), initially only imposed for convenience in order to derive the desired eigenfunctions, impose no actual loss of generality. By expanding the elliptic functions near the end-points of the intervals and using the regularization prescription implicit in $f$ described in the remark after Corollary 1 , that the limits of the functions $\lim _{s \rightarrow 0} U_{s}^{k}(u)=\chi_{k}(u)$ yields a set of functions whose span is equal to the set of indicator functions $\left\{1_{\left(a_{j}, b_{j}\right)}(u)\right\}_{j=1, \ldots, p}$. Therefore, the orthogonality relation implies that the $p$ eigenfunctions $\left\{V_{s}^{k}(u)\right\}_{k=1, \ldots, p}$ are already complete, because any eigenfunction must satisfy (166).

Then a similar analysis as in the case of one interval, expanding the bounded operator $F^{+}(s)$ on $\mathcal{K}_{A}$ in the generalized basis $U_{s}^{k}, V_{s}^{k}$ of $\Sigma_{A}$, gives again (164) with $H_{A}=$ $\int_{A \times A} k_{A}(u, v) j(u) j(v) \mathrm{d} u \mathrm{~d} v$, where

$$
\sum_{k=1}^{p} \int_{-\infty}^{\infty} \mathrm{d} s|s| U_{s}^{k}(u) \overline{U_{s}^{k}(v)}=: k_{A}(u, v)
$$

now. Again, we refrain here form analyzing in detail the remaining integrals.

\section{Conclusion}

In this work we have studied the modular flows of multi-component regions in chiral CFTs using methods from complex analysis. Our main tool was the KMS-condition built into modular theory, which we have combined with input from CFT such as locality and analyticity. The main general results concern matrix elements of the modular operator of the general type $\langle\Omega \mid \phi(x) f(\Delta) \phi(y) \Omega\rangle$, where $f$ are various functions such as ln, resolvents, complex powers, and $\phi$ a primary field. The states $|\Omega\rangle$ studied in this work were the vacuum and Gibbs (thermal) states. In some cases, our results for the matrix elements can be expressed in terms of integral equations of Cauchy-type [34,35], see e.g. Corollaries $1,2$.

Solving these types of equations was possible in a number of examples, such as free fermions and the $U(1)$-current. To keep the paper at a reasonable length, we have not carried out in this work all the resulting integrations, which would be needed if one is interested in more explicit answers. This would be possible. In the examples considered, we were also able to explain the relationship with known results in the literature $[10,11,15,16]$, and also with certain general, but formal (in the QFT context), 
Table 1. Periodicity properties, with $M=e^{-i \pi \tau} e^{-2 \pi i u}$

\begin{tabular}{lllll}
\hline & $\vartheta_{1}$ & $\vartheta_{2}$ & $\vartheta_{3}$ & $\vartheta_{4}$ \\
\hline$\vartheta_{i}(u+1) / \vartheta_{i}(u)$ & -1 & -1 & 1 & 1 \\
$\vartheta_{i}(u+\tau) / \vartheta_{i}(u)$ & $-M$ & $M$ & $M$ & $-M$ \\
\hline
\end{tabular}

methods known specifically for such free field theories, see [13,14]. In this sense, our methods also serve to make these formal approaches rigorous.

Our analysis is, in principle, not limited to such free CFTs, and in fact e.g., Corollaries 1,2, refer to general primary fields in an arbitrary chiral CFT, subject only to certain standard assumptions [40]. It would be interesting to study the implications of these general results further, which we leave for future work [41-44].

Acknowledgments Part of this work was carried out while I was visiting IHES, Paris (March 2019), and Instituto di Atomico Balseiro, Bariloche (March 2018). It is a pleasure to thank these institutions for hospitality and for financially supporting those visits. I am grateful to the Max-Planck Society for supporting the collaboration between MPI-MiS and Leipzig U., grant Proj. Bez. M.FE.A.MATN0003. Discussions with H. Casini, P. Fries, M. Huerta, F. Otto, and S. Del Vecchio are gratefully acknowledged.

Funding Open Access funding enabled and organized by Universität Leipzig (1039).

Open Access This article is licensed under a Creative Commons Attribution 4.0 International License, which permits use, sharing, adaptation, distribution and reproduction in any medium or format, as long as you give appropriate credit to the original author(s) and the source, provide a link to the Creative Commons licence, and indicate if changes were made. The images or other third party material in this article are included in the article's Creative Commons licence, unless indicated otherwise in a credit line to the material. If material is not included in the article's Creative Commons licence and your intended use is not permitted by statutory regulation or exceeds the permitted use, you will need to obtain permission directly from the copyright holder. To view a copy of this licence, visit http://creativecommons.org/licenses/by/4.0/.

Publisher's Note Springer Nature remains neutral with regard to jurisdictional claims in published maps and institutional affiliations.

\section{A. Conventions for Elliptic Functions}

Our conventions for the $\vartheta$-functions are:

$$
\begin{aligned}
& \vartheta_{1}(u ; \tau)=\sum_{n \in \mathbb{Z}}(-1)^{n-\frac{1}{2}} e^{i \pi \tau\left(n+\frac{1}{2}\right)^{2}+2 \pi i\left(n+\frac{1}{2}\right) u} \\
& \vartheta_{2}(u ; \tau)=\sum_{n \in \mathbb{Z}} e^{i \pi \tau\left(n+\frac{1}{2}\right)^{2}+2 \pi i\left(n+\frac{1}{2}\right) u} \\
& \vartheta_{3}(u ; \tau)=\sum_{n \in \mathbb{Z}} e^{i \pi \tau n^{2}+2 \pi i n u}, \\
& \vartheta_{4}(u ; \tau)=\sum_{n \in \mathbb{Z}}(-1)^{n} e^{i \pi \tau n^{2}+2 \pi i n u} .
\end{aligned}
$$

The $\wp$-function is given by

$$
\wp(u ; \tau)=\sum_{(m, n) \neq(0,0)}\left(\frac{1}{(u+m+\tau n)^{2}}-\frac{1}{(m+\tau n)^{2}}\right) .
$$


The Weierstrass $\zeta$-function is a function defined so that $\zeta^{\prime}(u, \tau)=-\wp(u ; \tau)$. Explicitly,

$$
\zeta(u ; \tau)=\frac{1}{u}+\sum_{(m, n) \neq(0,0)}\left(\frac{1}{u+m+\tau n}-\frac{1}{m+\tau n}+\frac{u}{(m+\tau n)^{2}}\right) .
$$

It has the periodicity $\zeta(u+1)=\zeta(u)+\eta_{1}, \zeta(u+\tau)=\zeta(u)+\eta_{2}$.

\section{References}

1. Longo, R., Xu, F.: Comment on the Bekenstein bound. J. Geom. Phys. 130, 113 (2018)

2. Bousso, R., Fisher, Z., Leichenauer, S., Wall, A.C.: Quantum focusing conjecture. Phys. Rev. D 93(6), 064044 (2016)

3. Bousso, R., Fisher, Z., Koeller, J., Leichenauer, S., Wall, A.C.: Proof of the quantum null energy condition. Phys. Rev. D 93(2), 024017 (2016)

4. Casini, H., Huerta, M.: A c-theorem for the entanglement entropy. J. Phys. A 40, 7031 (2007)

5. Rangamani, M., Takayanagi, T.: Holographic Entanglement Entropy, Springer Lecture Notes in Physics (2017)

6. Takesaki, M.: Theory of Operator Algebras I-III. Springer, Berlin (2003)

7. Bisognano, J.J., Wichmann, E.H.: On the duality condition for quantum fields. J. Math. Phys. 17, 303 (1976)

8. Hislop, P.D., Longo, R.: Modular structure of the local algebras associated with the free massless scalar field theory. Commun. Math. Phys. 84, 71 (1982)

9. Brunetti, R., Guido, D., Longo, R.: Modular structure and duality in conformal quantum field theory. Commun. Math. Phys. 156, 201 (1993)

10. Casini, H., Huerta, M.: Reduced density matrix and internal dynamics for multicomponent regions. Class. Quant. Grav. 26, 185005 (2009)

11. Arias, R.E., Casini, H., Huerta, M., Pontello, D.: Entropy and modular Hamiltonian for a free chiral scalar in two intervals. Phys. Rev. D 98(12), 125008 (2018)

12. Haag, R., Hugenholtz, N.M., Winnink, M.: On the equilibrium states in quantum statistical mechanics. Commun. Math. Phys. 5, 215 (1967)

13. Peschel, I.: Calculation of reduced density matrices from correlation functions. J. Phys. A 36, L205 (2003). https://doi.org/10.1088/0305-4470/36/14/101

14. Peschel, I., Eisler, V.: Reduced density matrices and entanglement entropy in free lattice models. J. Phys. A Math. Theor. 42, 504003 (2009)

15. Blanco, D., Perez-Nadal, G.: Modular Hamiltonian of a chiral fermion on the torus. Phys. Rev. D 100(2), 025003 (2019). https://doi.org/10.1103/PhysRevD.100.025003

16. Fries, P., Reyes, I.A.: The entanglement spectrum of chiral fermions on the torus. Phys. Rev. Lett. 123(21), 211603 (2019). https://doi.org/10.1103/PhysRevLett.123.211603

17. Bratteli, O., Robinson, D.W.: Operator Algebras and Quantum Statistical Mechanics I. Springer, Berlin (1987)

18. Bratteli, O., Robinson, D.W.: Operator Algebras and Quantum Statistical Mechanics II. Springer, Berlin (1997)

19. Hollands, S., Sanders, K.: Entanglement measures and their properties in quantum field theory, Springer Briefs in Mathematical Physics (2018)

20. Witten, E.: Notes on Some Entanglement Properties of Quantum Field Theory, arXiv:1803.04993 [hep-th]

21. Haag, R.: Local Quantum Physics: Fields, Particles. Algebras. Springer, Berlin (1992)

22. Gabbiani, F., Fröhlich, J.: Operator algebras and conformal field theories. Commun. Math. Phys. 155, 569-640 (1993)

23. Carpi, S., Kawahigashi, Y., Longo, R., Weiner, M.: From vertex operator algebras to conformal nets and back, arXiv:1503.01260 [math.OA]

24. Fredenhagen, K.: On the modular structure of local algebras of observables. Commun. Math. Phys. 97, 79-89 (1985)

25. Buchholz, D., Fredenhagen, K., D’Antoni, C.: The universal structure of local algebras. Commun. Math. Phys. 111, 123 (1987)

26. Buchholz, D., Mack, G., Todorov, I.: The current algebra on the circle as a germ of local field theories. Nucl. Phys. Proc. Suppl. 5B, 20 (1988)

27. Bischoff, M., Tanimoto, Y.: Construction of wedge-local nets of observables through Longo-Witten endomorphisms II. Commun. Math. Phys. 317, 667 (2013). https://doi.org/10.1007/s00220-012-1593-X

28. Buchholz, D., Schulz-Mirbach, H.: Haag duality in conformal quantum field theory. Rev. Math. Phys. 2, 105 (1990) 
29. Goodman, R., Wallach, N.R.: Structure and unitary cocycle representations of loop groups and the group of diffeomorphisms of the circle. J. Reine Angew. Math. 347, 69-133 (1984)

30. Goodman, R., Wallach, N.R.: Projective unitary positive-energy representations of $\operatorname{Diff}\left(S^{1}\right)$. J. Funct. Anal. 63(3), 299-321 (1985)

31. Lang, S.: $S L_{2}(\mathbb{R} \mathbb{R})$. Springer, Berlin (1985)

32. Rehren, K.H., Tedesco, G.: Multilocal fermionization. Lett. Math. Phys. 103, 19 (2013)

33. Araki, H.: On quasifree states of the CAR and Bogoliubov automorphisms. Publ. RIMS Kyoto Univ. 6, 385-442 (1970)

34. Muskhelishvili, N.I.: Singular Integral Equations. Wolters-Noordhoff Publishing, Groningen (1958)

35. Gakhov, FD: Boundary Value Problems, Dover (1990), reprint from 1966 edition in Pergamon Press

36. Longo, R., Martinetti, P., Rehren, K.H.: Geometric modular action for disjoint intervals and boundary conformal field theory. Rev. Math. Phys. 22, 331 (2010)

37. Accardi, L., Cecchini, C.: Conditional expectations in von Neumann Algebras and a Theorem of Takesaki. J. Funct. Anal. 45, 245-273 (1982)

38. Hollands, S., Wald, R.M.: Existence of local covariant time ordered products of quantum fields in curved space-time. Commun. Math. Phys. 231, 309 (2002). https://doi.org/10.1007/s00220-002-0719-y

39. Itzykson, C., Zuber, J.B.: Two-dimensional conformal invariant theories on a torus. Nucl. Phys. B $\mathbf{2 7 5}$, 580 (1986). https://doi.org/10.1016/0550-3213(86)90576-6

40. S. Hollands, in progress

41. Araki, H.: Relative Hamiltonian for faithful normal states of a von Neumann algebra. Publ. RIMS Kyoto Univ. 9, 165-209 (1973)

42. Araki, H.: Relative entropy of states of von Neumann algebras. I. Publ. RIMS Kyoto Univ. 11, 809-833 (1976)

43. Araki, H.: Relative entropy of states of von Neumann algebras. II. Publ. RIMS Kyoto Univ. 13, 173-192 (1977)

44. Fredenhagen, K., Rehren, K.H., Schroer, B.: Superselection sectors with braid group statistics and exchange algebras. 2. Geometric aspects and conformal covariance. Rev. Math. Phys. 4(spec01), 113 (1992)

Communicated by Y. Kawahigashi 